\title{
Correlations between Fukui Indices and Reactivity Descriptors based on the Sanderson's Principle
}

\author{
Jesús Sánchez-Márquez*1. \\ ${ }^{1}$ Departamento de Química-Física, Facultad de Ciencias, Campus Universitario \\ Río San Pedro, Universidad de Cádiz, 11510 Puerto Real, Cádiz, Spain \\ *To whom correspondence should be addressed. E-mail: jesus.sanchezmarquez@uca.es
}

\section{SUPPORTING INFORMATION}

\section{FIGURES}

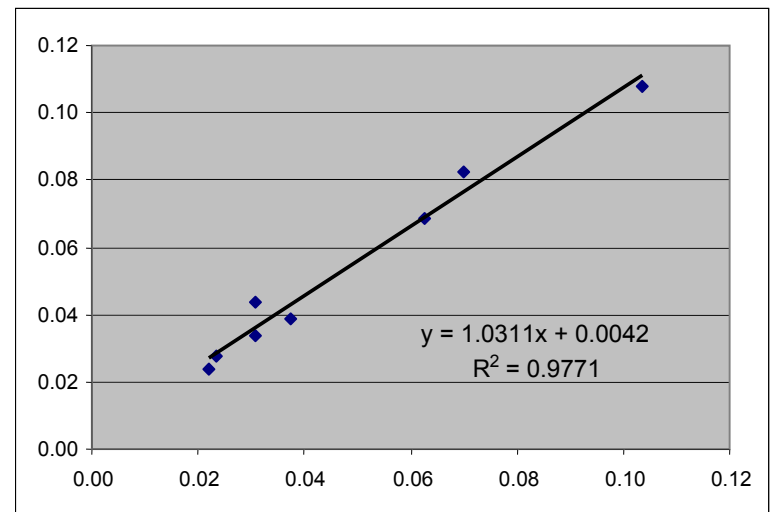

Figure S1. $\Delta N_{A}^{\max }$ values calculated with the first model (Eq. 10) vs. $\Delta N_{A}^{\max }$ calculated with the second model (Eq. 25) for the molecule $\mathrm{CH}_{2} \mathrm{CHCHO}$. The values of the two parameters have been obtained using the Hirshfeld population analysis. 


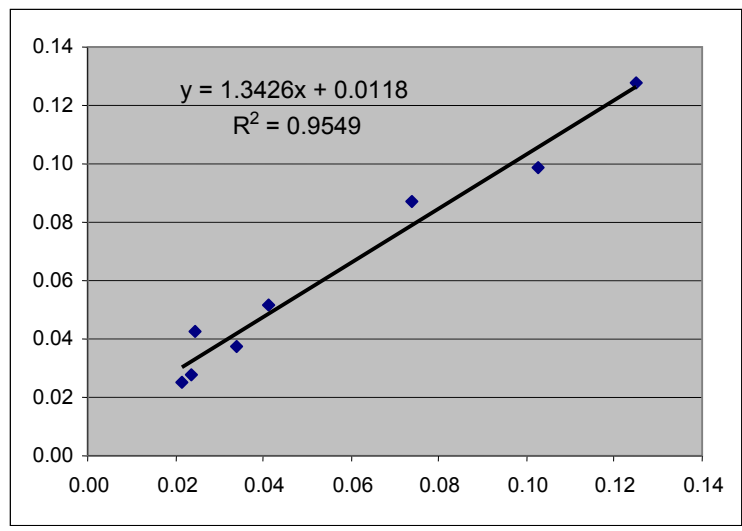

Figure S2. $\Delta N_{A}^{\max }$ values calculated with the first model (Eq. 10) vs. $\Delta N_{A}^{\max }$ calculated with the second model (Eq. 25) for the molecule $\mathrm{CH}_{2} \mathrm{CHNO}_{2}$. The values of the two parameters have been obtained using the Hirshfeld population analysis.

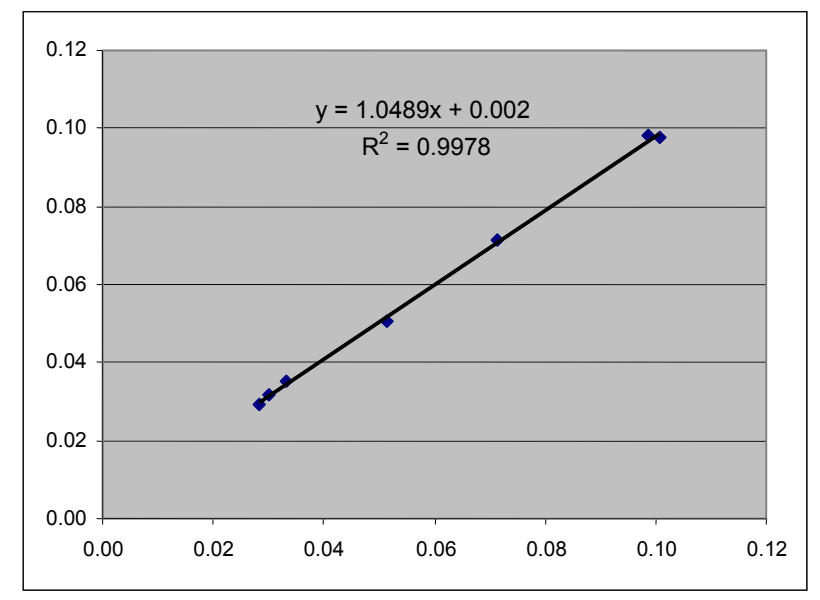

Figure S3. $\Delta N_{A}^{\max }$ values calculated with the first model (Eq. 10) vs. $\Delta N_{A}^{\max }$ calculated with the second model (Eq. 25) for the molecule $\mathrm{CH}_{2} \mathrm{CHCN}$. The values of the two parameters have been obtained using the Hirshfeld population analysis. 


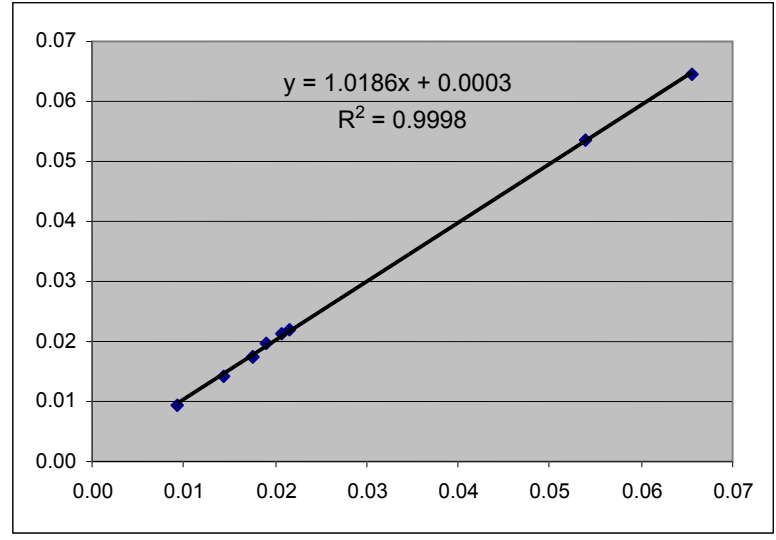

Figure S4. $\Delta N_{A}^{\max }$ values calculated with the first model (Eq. 10) vs. $\Delta N_{A}^{\max }$ calculated with the second model (Eq. 25) for the molecule $\mathrm{CH}_{2} \mathrm{CHCH}_{3}$. The values of the two parameters have been obtained using the Hirshfeld population analysis.

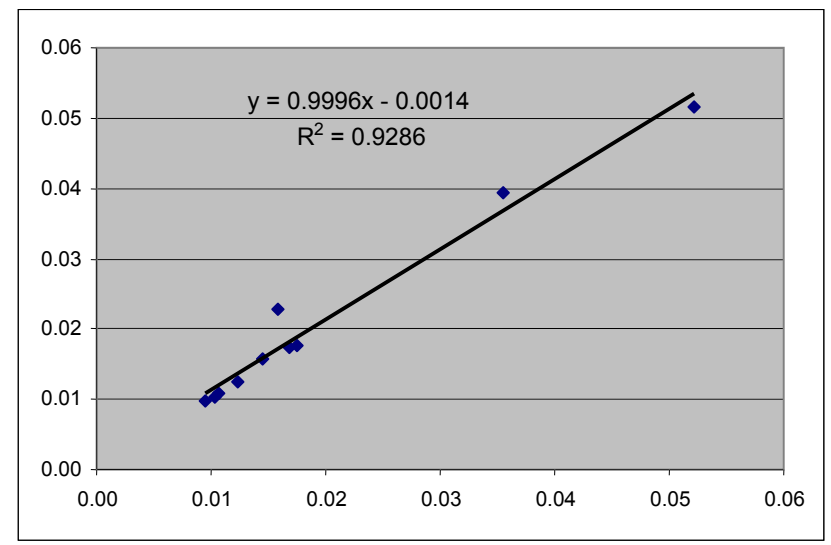

Figure S5. $\Delta N_{A}^{\max }$ values calculated with the first model (Eq. 10) vs. $\Delta N_{A}^{\max }$ calculated with the second model (Eq. 25) for the molecule $\mathrm{CH}_{2} \mathrm{CHOCH}_{3}$. The values of the two parameters have been obtained using the Hirshfeld population analysis. 


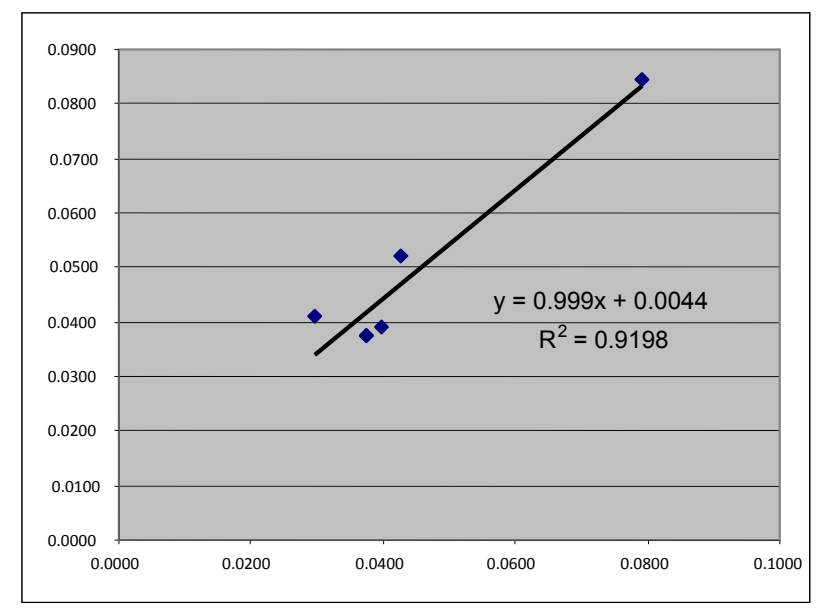

Figure S6. $\Delta N_{A}^{\max }$ values calculated with the first model (Eq. 10) vs. $\Delta N_{A}^{\max }$ calculated with the second model (Eq. 25) for the molecule $\mathrm{CH}_{2} \mathrm{CHCl}$. The values of the two parameters have been obtained using the population analysis of QTAIM.

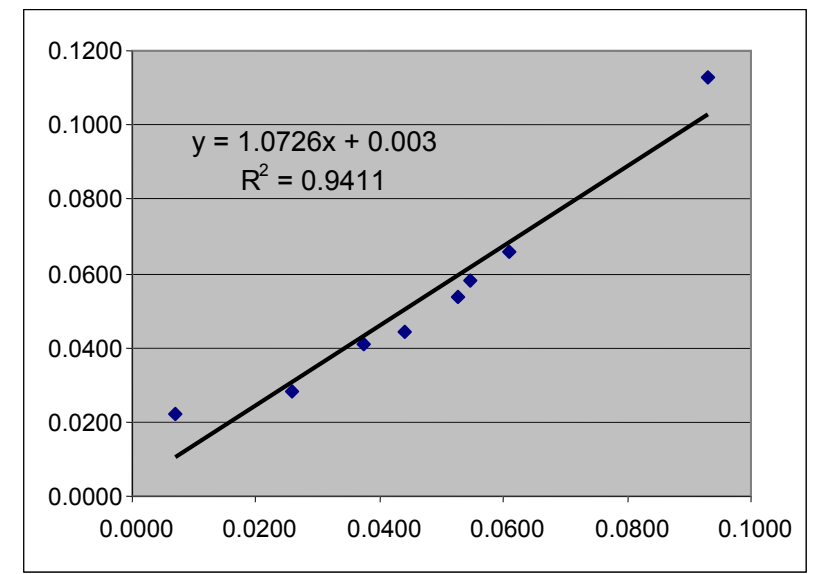

Figure S7. $\Delta N_{A}^{\max }$ values calculated with the first model (Eq. 10) vs. $\Delta N_{A}^{\max }$ calculated with the second model (Eq. 25) for the molecule $\mathrm{CH}_{2} \mathrm{CHCHO}$. The values of the two parameters have been obtained using the population analysis of QTAIM.

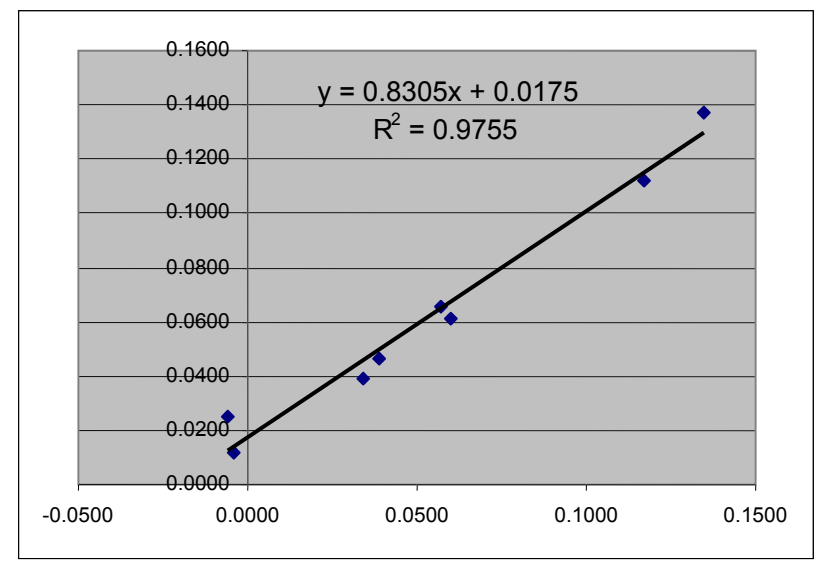

Figure S8. $\Delta N_{A}^{\max }$ values calculated with the first model (Eq. 10) vs. $\Delta N_{A}^{\max }$ calculated with the second model (Eq. 25) for the molecule $\mathrm{CH}_{2} \mathrm{CHNO}_{2}$. The values of the two parameters have been obtained using the population analysis of QTAIM. 


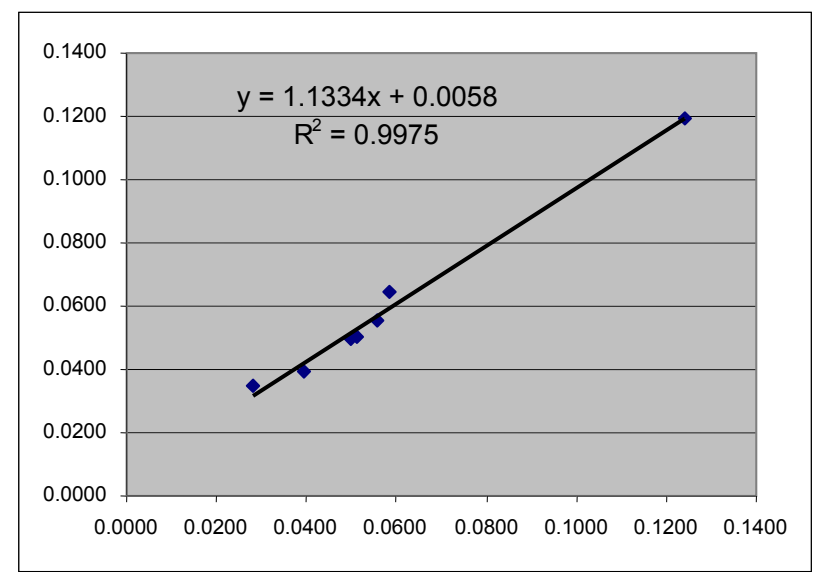

Figure S9. $\Delta N_{A}^{\max }$ values calculated with the first model (Eq. 10) vs. $\Delta N_{A}^{\max }$ calculated with the second model (Eq. 25) for the molecule $\mathrm{CH}_{2} \mathrm{CHCN}$. The values of the two parameters have been obtained using the population analysis of QTAIM.

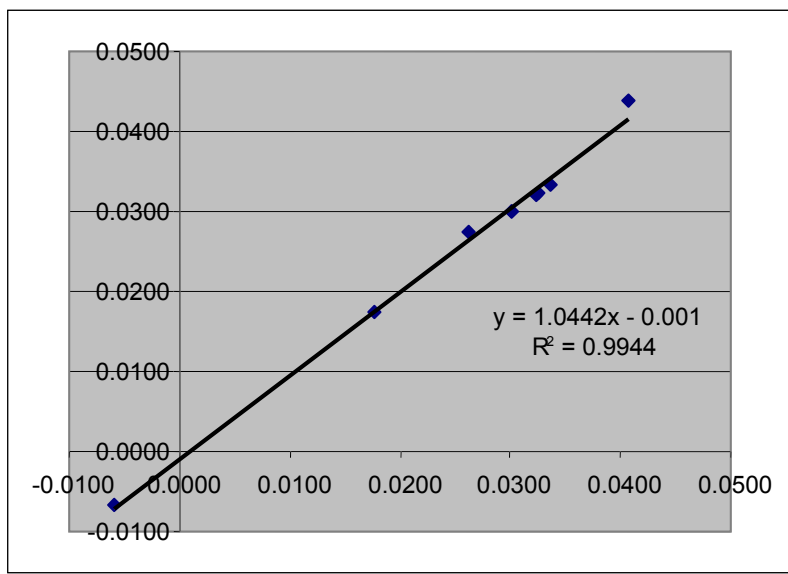

Figure S10. $\Delta N_{A}^{\max }$ values calculated with the first model (Eq. 10) vs. $\Delta N_{A}^{\max }$ calculated with the second model (Eq. 25) for the molecule $\mathrm{CH}_{2} \mathrm{CHCH}_{3}$. The values of the two parameters have been obtained using the population analysis of QTAIM.

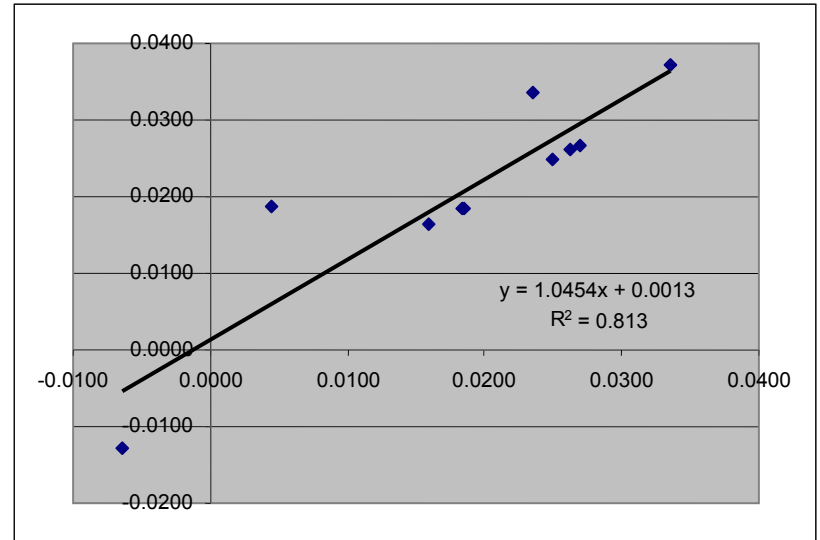

Figure S11. $\Delta N_{A}^{\max }$ values calculated with the first model (Eq. 10) vs. $\Delta N_{A}^{\max }$ calculated with the second model (Eq. 25) for the molecule $\mathrm{CH}_{2} \mathrm{CHOCH}_{3}$. The values of the two parameters have been obtained using the population analysis of QTAIM. 


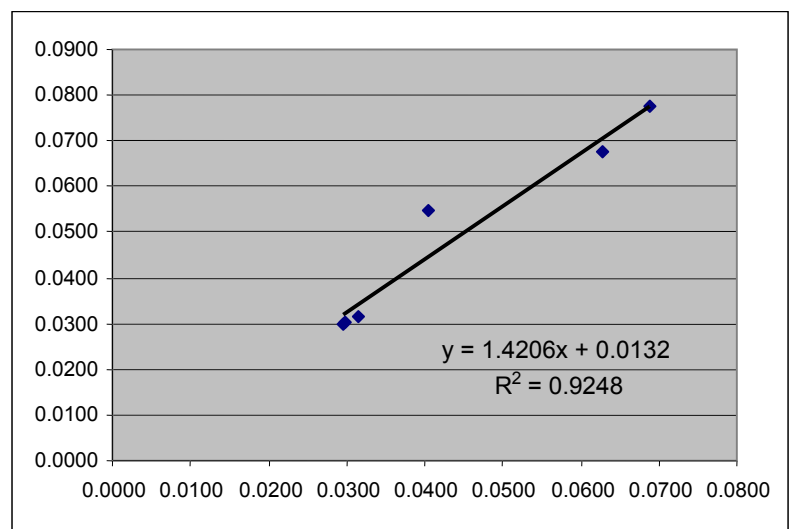

Figure S12. $\Delta N_{A}^{\max }$ values calculated with the first model (Eq. 10) vs. $\Delta N_{A}^{\max }$ calculated with the second model (Eq. 25) for the molecule $\mathrm{CH}_{2} \mathrm{CHCl}$. The values of the two parameters have been obtained using the Mulliken population analysis.

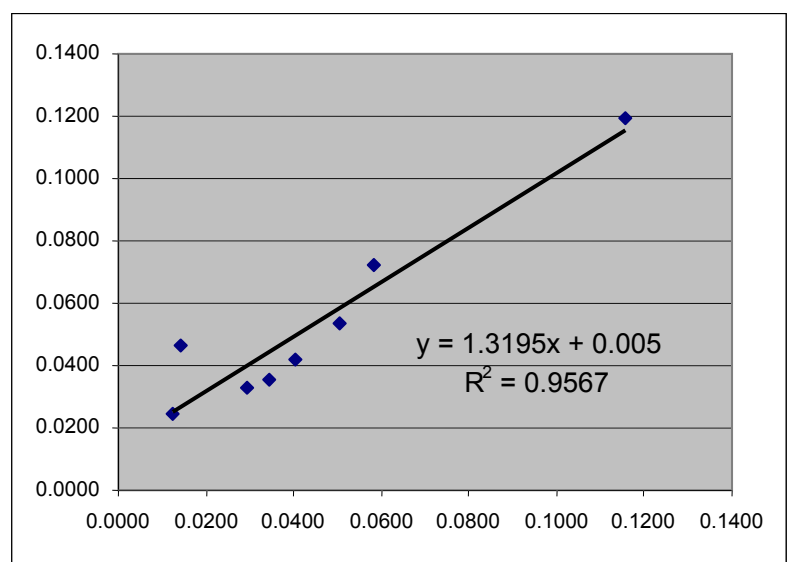

Figure S13. $\Delta N_{A}^{\max }$ values calculated with the first model (Eq. 10) vs. $\Delta N_{A}^{\max }$ calculated with the second model (Eq. 25) for the molecule $\mathrm{CH}_{2} \mathrm{CHCHO}$. The values of the two parameters have been obtained using the Mulliken population analysis.

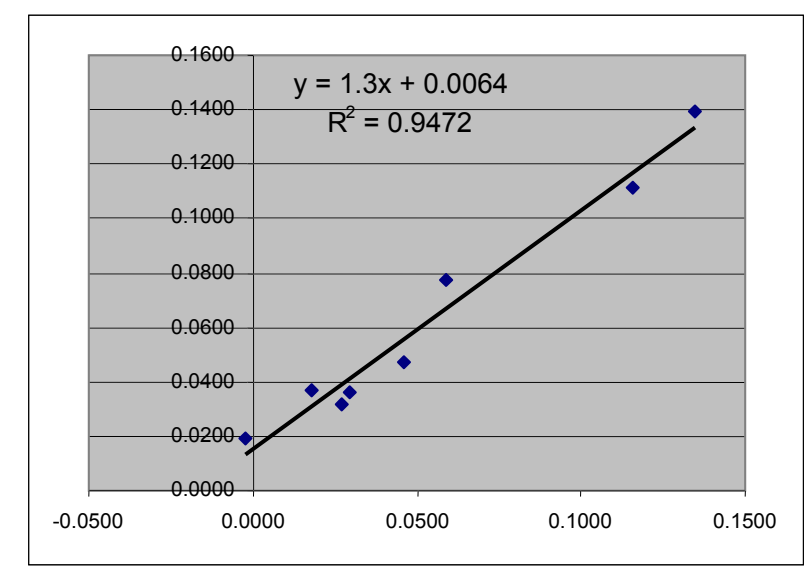

Figure S14. $\Delta N_{A}^{\max }$ values calculated with the first model (Eq. 10) vs. $\Delta N_{A}^{\max }$ calculated with the second model (Eq. 25) for the molecule $\mathrm{CH}_{2} \mathrm{CHNO}_{2}$. The values of the two parameters have been obtained using the Mulliken population analysis. 


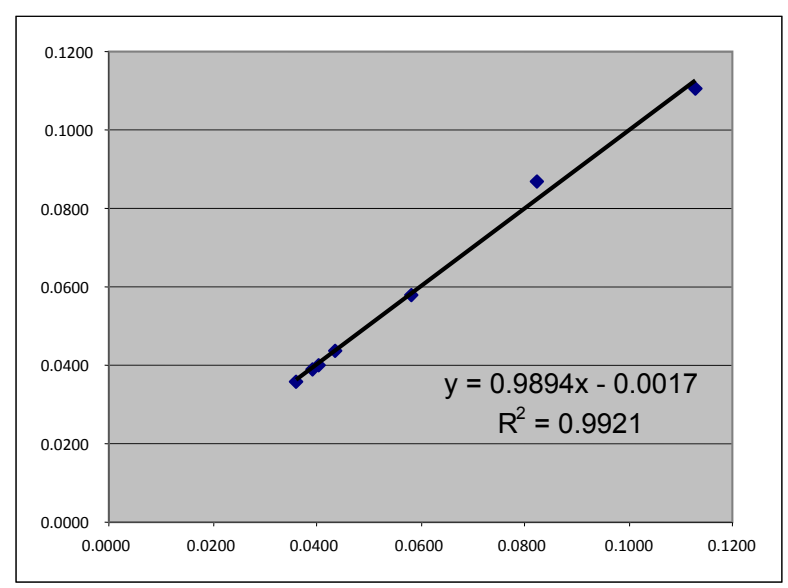

Figure S15. $\Delta N_{A}^{\max }$ values calculated with the first model (Eq. 10) vs. $\Delta N_{A}^{\max }$ calculated with the second model (Eq. 25) for the molecule $\mathrm{CH}_{2} \mathrm{CHCN}$. The values of the two parameters have been obtained using the Mulliken population analysis.

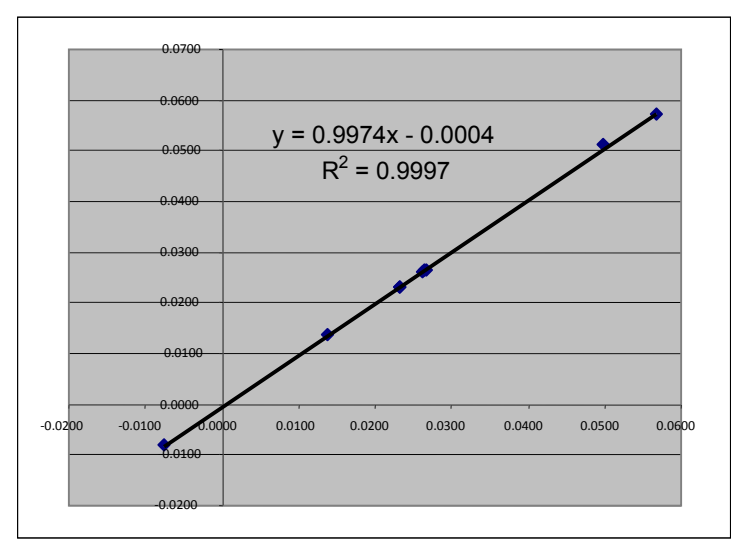

Figure S16. $\Delta N_{A}^{\max }$ values calculated with the first model (Eq. 10) vs. $\Delta N_{A}^{\max }$ calculated with the second model (Eq. 25) for the molecule $\mathrm{CH}_{2} \mathrm{CHCH}_{3}$. The values of the two parameters have been obtained using the Mulliken population analysis.

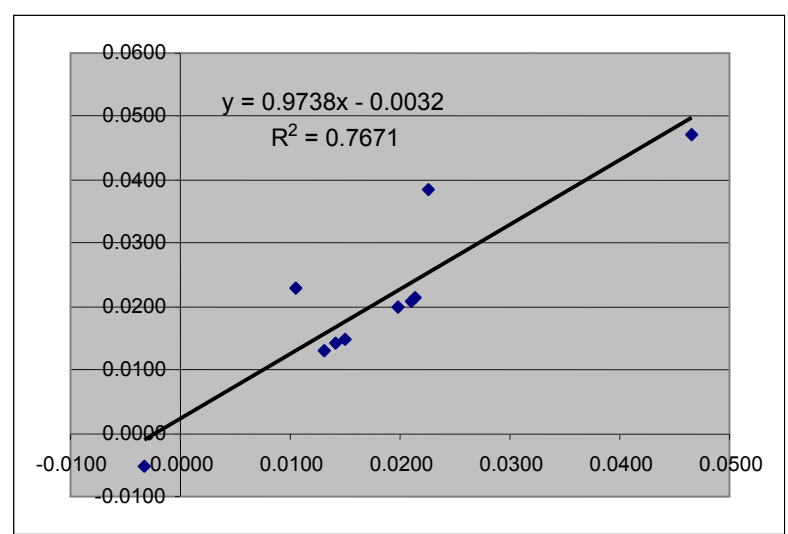

Figure S17. $\Delta N_{A}^{\max }$ values calculated with the first model (Eq. 10) vs. $\Delta N_{A}^{\max }$ calculated with the second model (Eq. 25) for the molecule $\mathrm{CH}_{2} \mathrm{CHOCH}_{3}$. The values of the two parameters have been obtained using the Mulliken population analysis. 


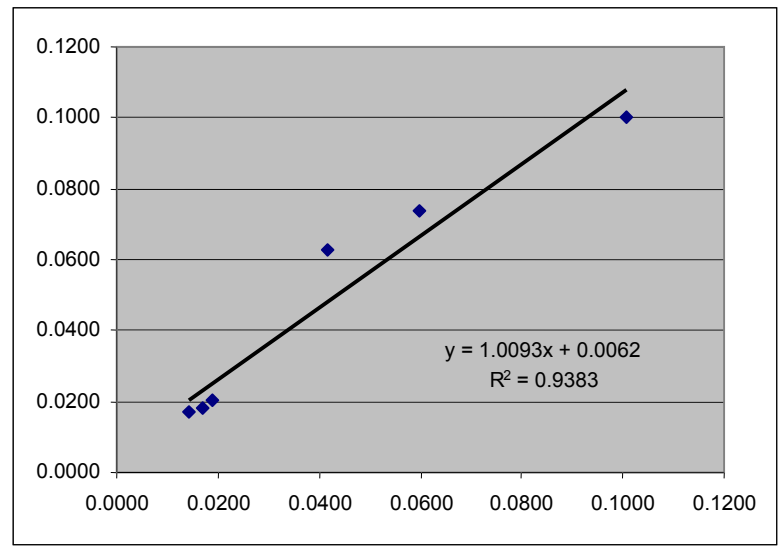

Figure S18. $\Delta N_{A}^{\max }$ values calculated with the first model (Eq. 10) vs. $\Delta N_{A}^{\max }$ calculated with the second model (Eq. 25) for the molecule $\mathrm{CH}_{2} \mathrm{CHCl}$. The values of the two parameters have been obtained using the Natural Population Analysis.

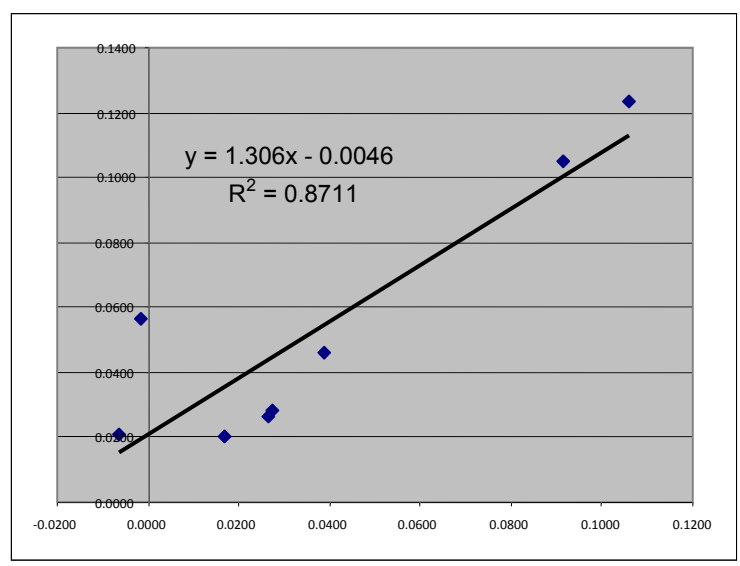

Figure S19. $\Delta N_{A}^{\max }$ values calculated with the first model (Eq. 10) vs. $\Delta N_{A}^{\max }$ calculated with the second model (Eq. 25) for the molecule $\mathrm{CH}_{2} \mathrm{CHCHO}$. The values of the two parameters have been obtained using the Natural Population Analysis.

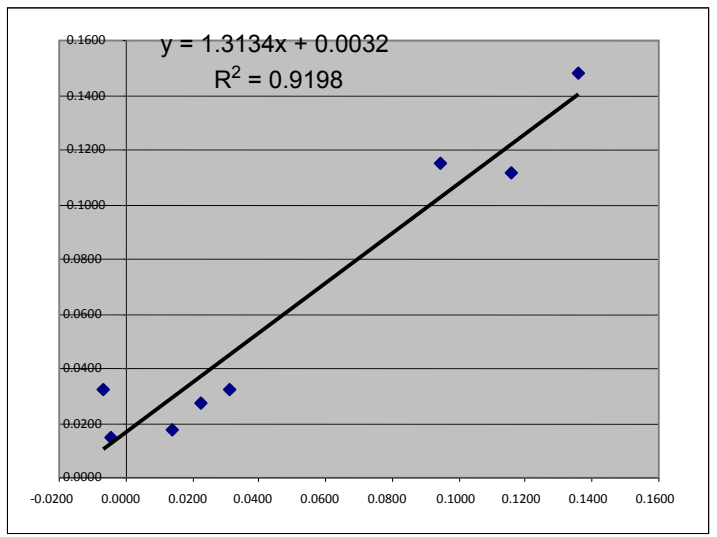

Figure S20. $\Delta N_{A}^{\max }$ values calculated with the first model (Eq. 10) vs. $\Delta N_{A}^{\max }$ calculated with the second model (Eq. 25) for the molecule $\mathrm{CH}_{2} \mathrm{CHNO}_{2}$. The values of the two parameters have been obtained using the Natural Population Analysis. 


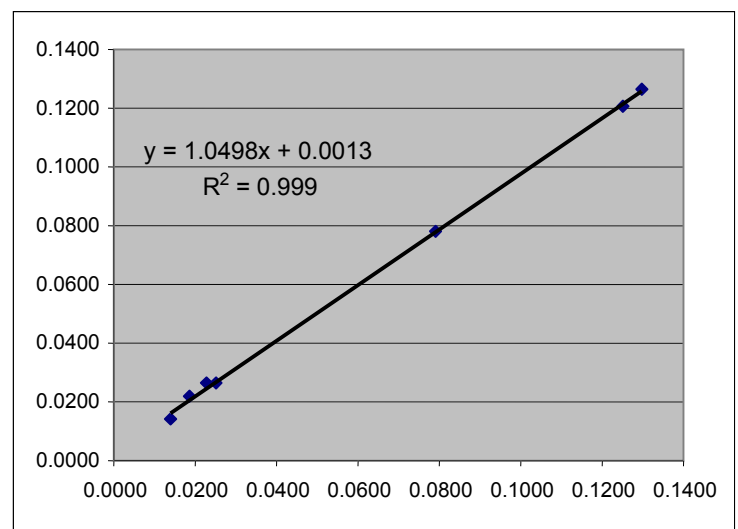

Figure S21. $\Delta N_{A}^{\max }$ values calculated with the first model (Eq. 10) vs. $\Delta N_{A}^{\max }$ calculated with the second model (Eq. 25) for the molecule $\mathrm{CH}_{2} \mathrm{CHCN}$. The values of the two parameters have been obtained using the Natural Population Analysis.

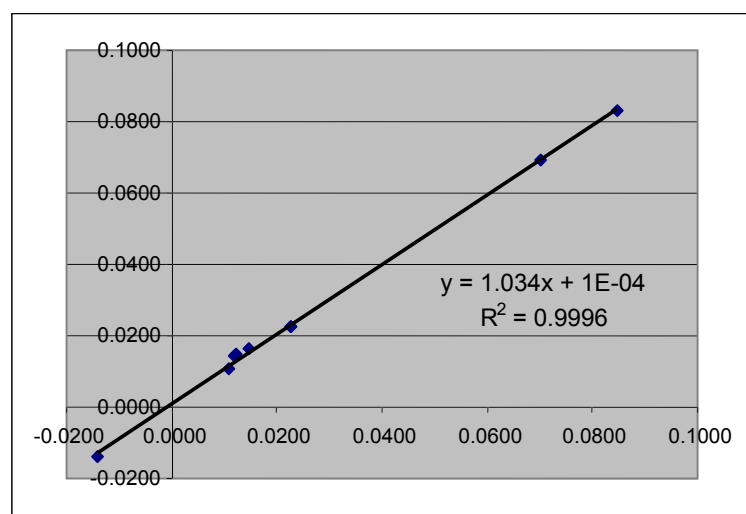

Figure S22. $\Delta N_{A}^{\max }$ values calculated with the first model (Eq. 10) vs. $\Delta N_{A}^{\max }$ calculated with the second model (Eq. 25) for the molecule $\mathrm{CH}_{2} \mathrm{CHCH}_{3}$. The values of the two parameters have been obtained using the Natural Population Analysis.

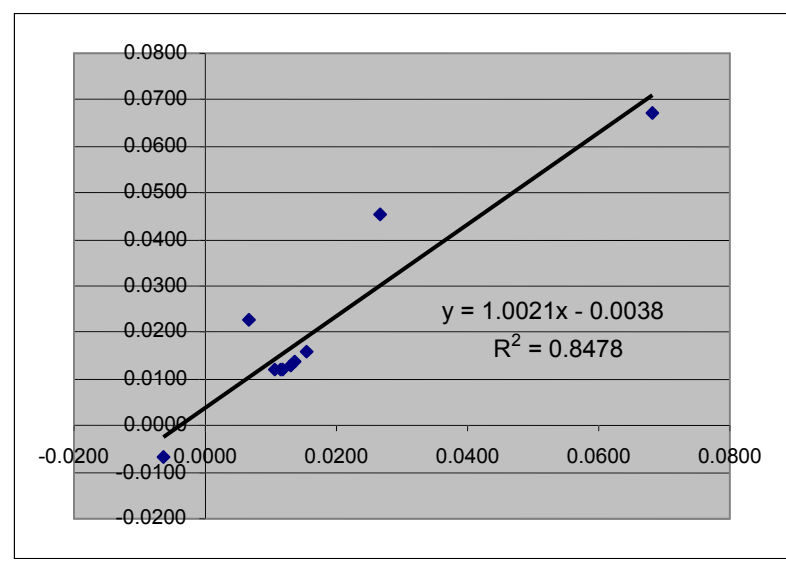

Figure S23. $\Delta N_{A}^{\max }$ values calculated with the first model (Eq. 10) vs. $\Delta N_{A}^{\max }$ calculated with the second model (Eq. 25) for the molecule $\mathrm{CH}_{2} \mathrm{CHOCH}_{3}$. The values of the two parameters have been obtained using the Natural Population Analysis. 


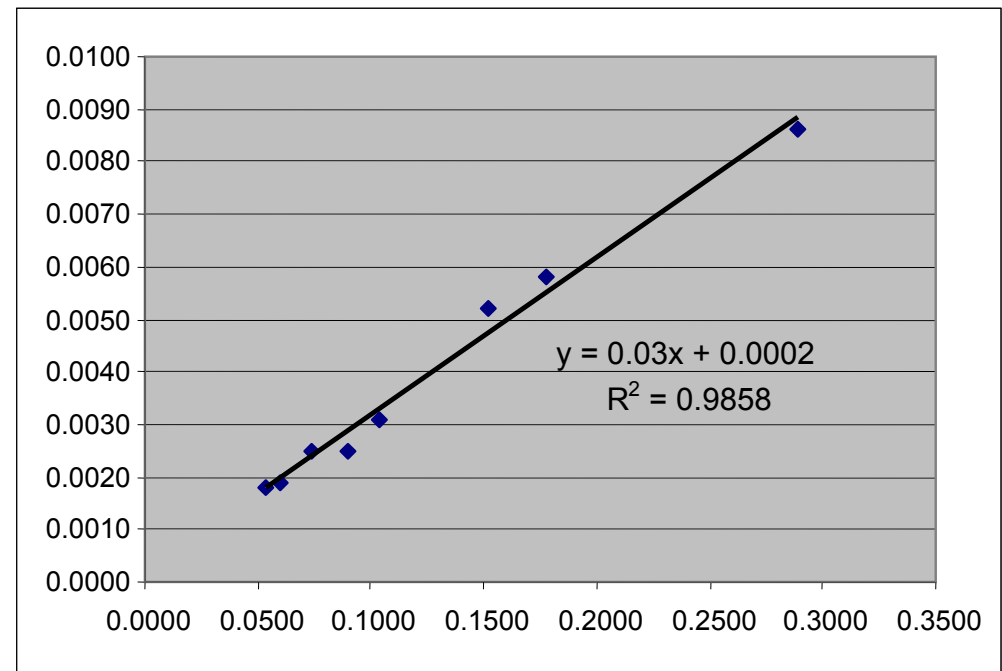

Figure S24. Linear regression $\omega_{A}$ (Eq. 11, first model) versus $f_{A}$ (Eq. 24, second model) for the molecule $\mathrm{CH}_{2} \mathrm{CHCHO}$. Both parameters have been obtained using the Hirshfeld population analysis.

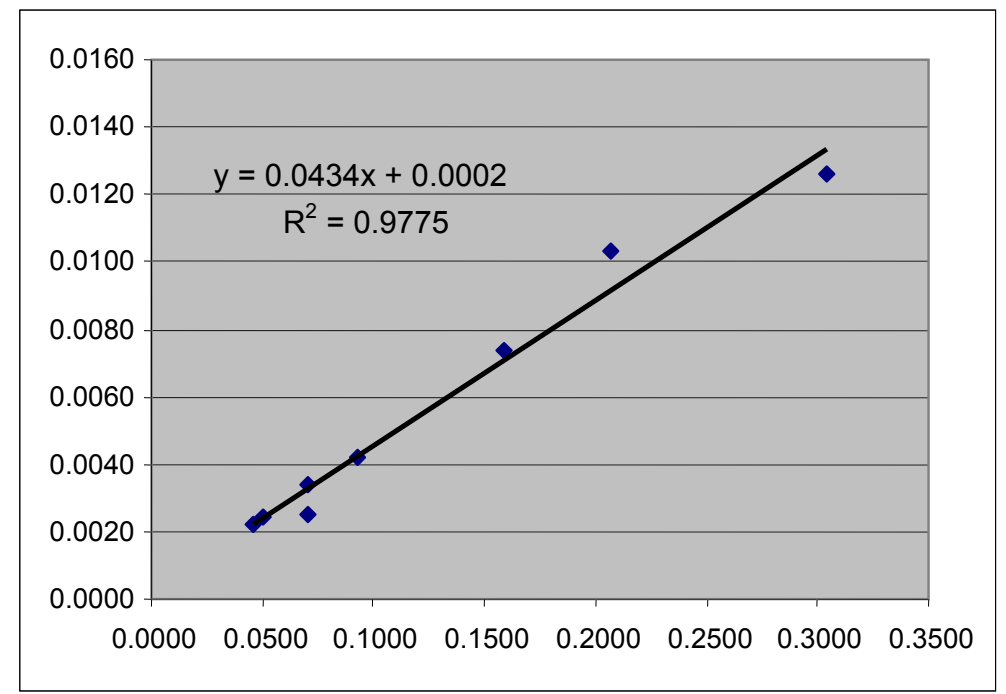

Figure S25. Linear regression $\omega_{A}$ (Eq. 11, first model) versus $f_{A}$ (Eq. 24, second model) for the molecule $\mathrm{CH}_{2} \mathrm{CHNO}_{2}$. Both parameters have been obtained using the Hirshfeld population analysis. 


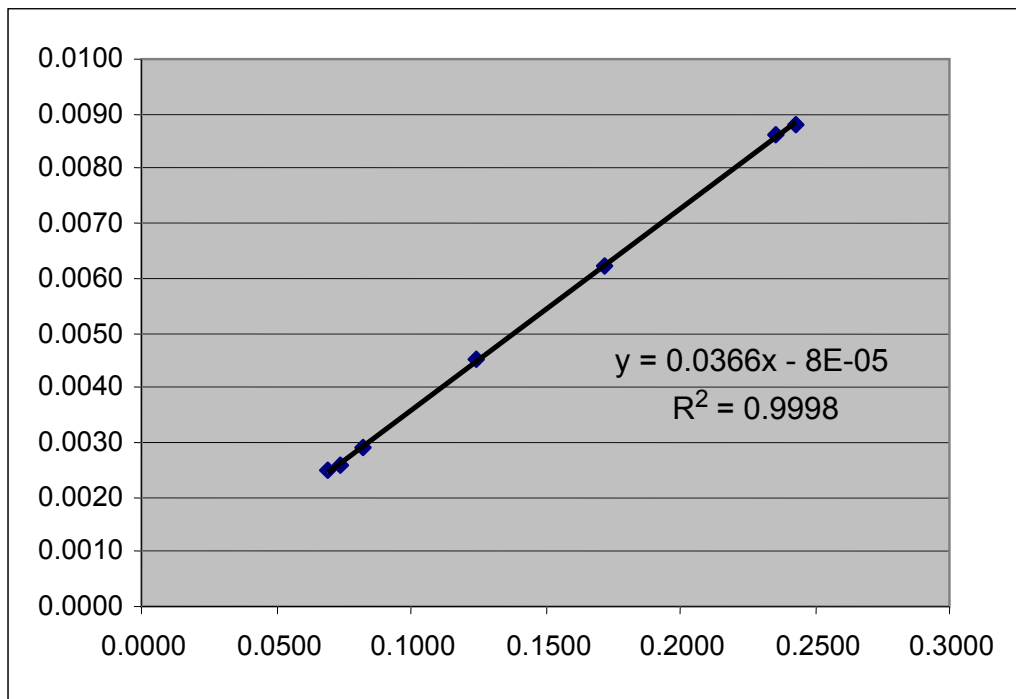

Figure S26. Linear regression $\omega_{A}$ (Eq. 11, first model) versus $f_{A}$ (Eq. 24, second model) for the molecule $\mathrm{CH}_{2} \mathrm{CHCN}$. Both parameters have been obtained using the Hirshfeld population analysis.

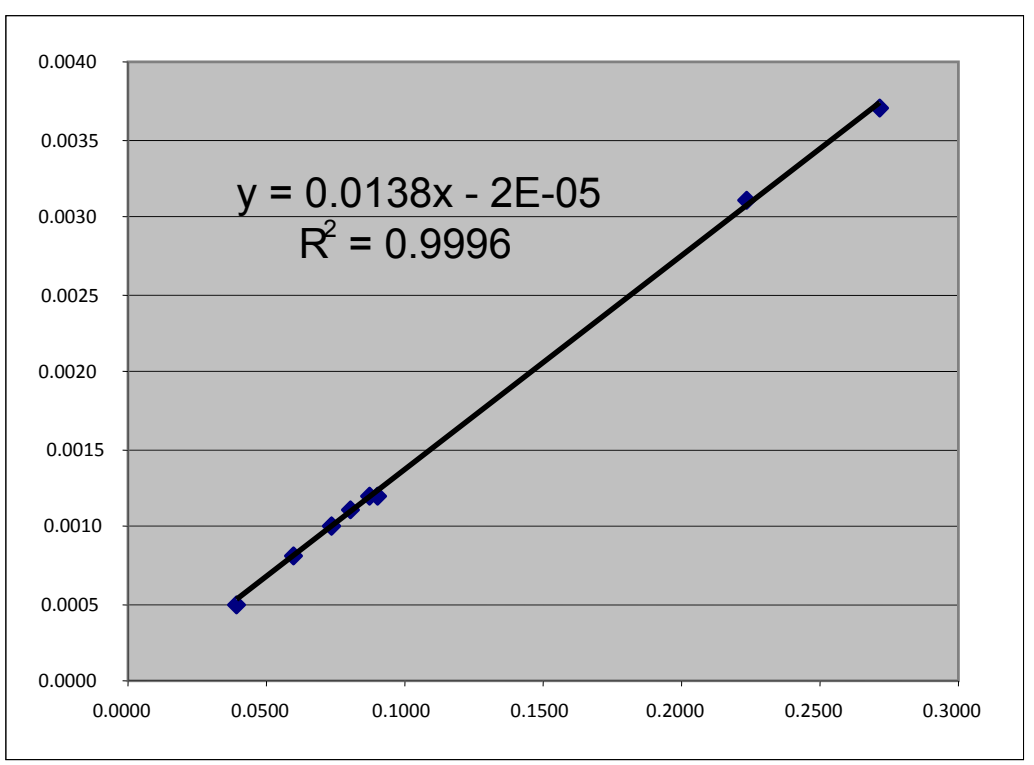

Figure S27. Linear regression $\omega_{A}$ (Eq. 11, first model) versus $f_{A}$ (Eq. 24, second model) for the molecule $\mathrm{CH}_{2} \mathrm{CHCH}_{3}$. Both parameters have been obtained using the Hirshfeld population analysis. 


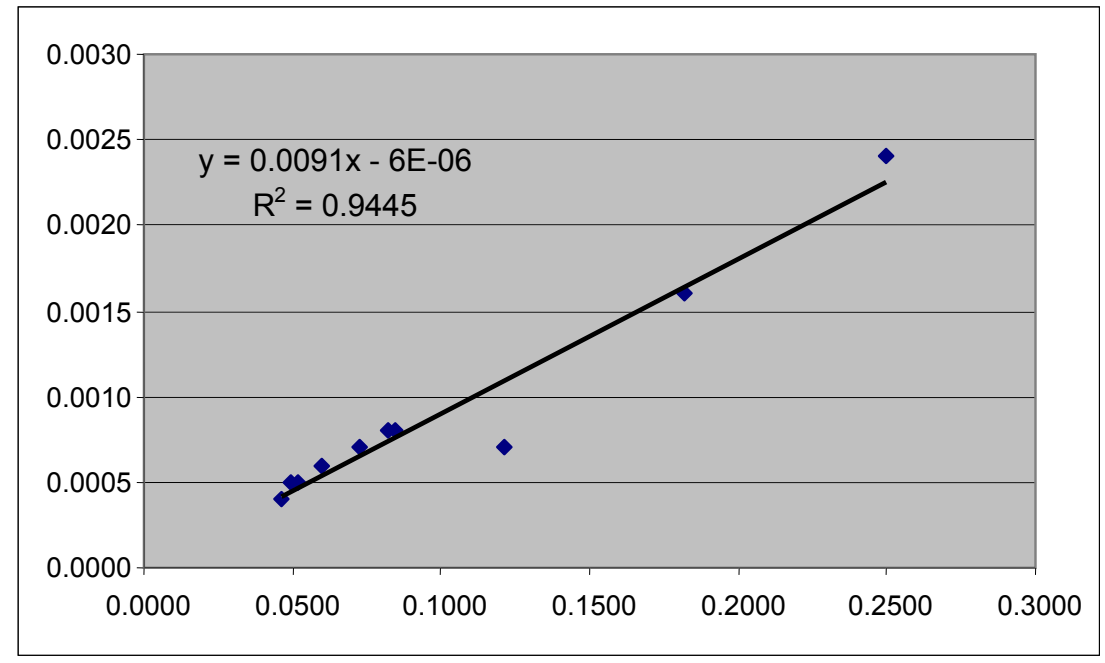

Figure S28. Linear regression $\omega_{A}$ (Eq. 11, first model) versus $f_{A}$ (Eq. 24, second model) for the molecule $\mathrm{CH}_{2} \mathrm{CHOCH}_{3}$. Both parameters have been obtained using the Hirshfeld population analysis.

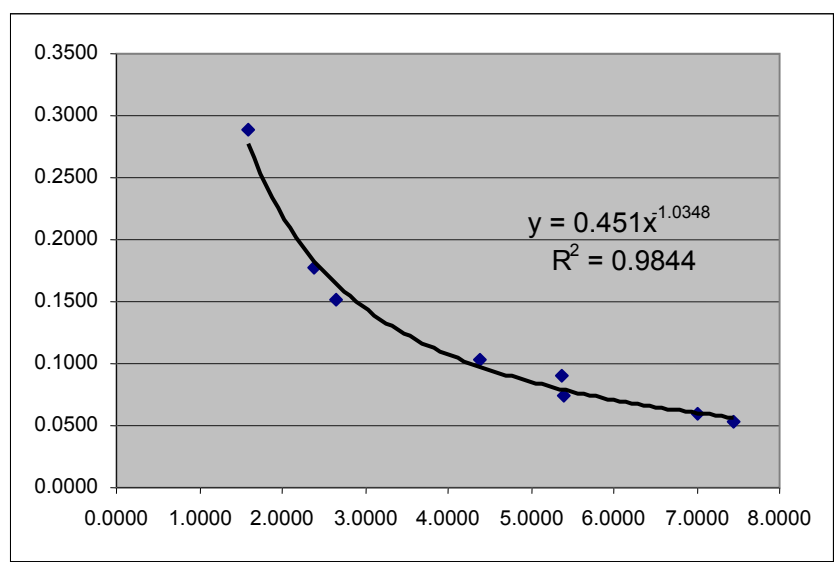

Figure S29. $f_{A}$ values calculated with the second model (Eq. 24) vs. $\eta_{A}$ parameter calculated with the first model (Eq. 8) for the molecule $\mathrm{CH}_{2} \mathrm{CHCHO}$. The values of the two parameters have been obtained using the Hirshfeld population analysis.

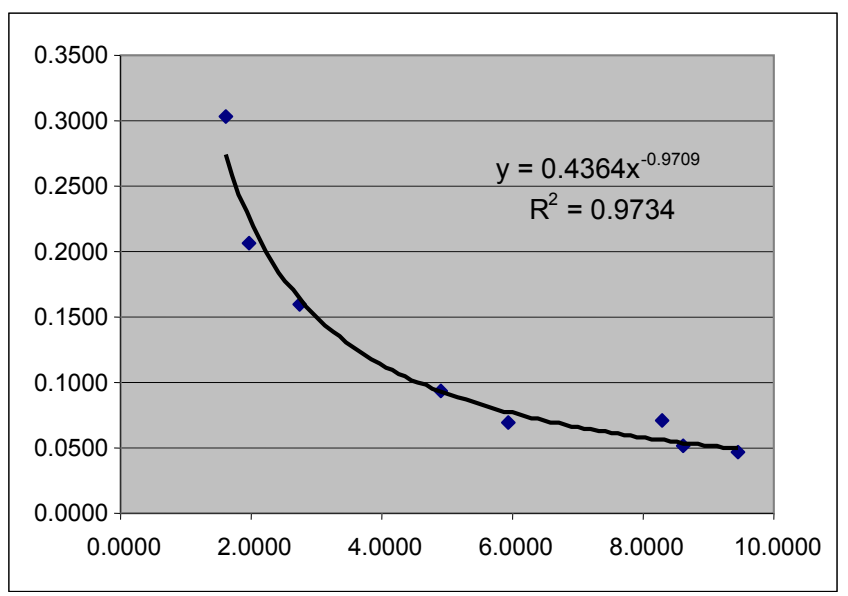


Figure S30. $f_{A}$ values calculated with the second model (Eq. 24) vs. $\eta_{A}$ parameter calculated with the first model (Eq. 8) for the molecule $\mathrm{CH}_{2} \mathrm{CHNO}_{2}$. The values of the two parameters have been obtained using the Hirshfeld population analysis.

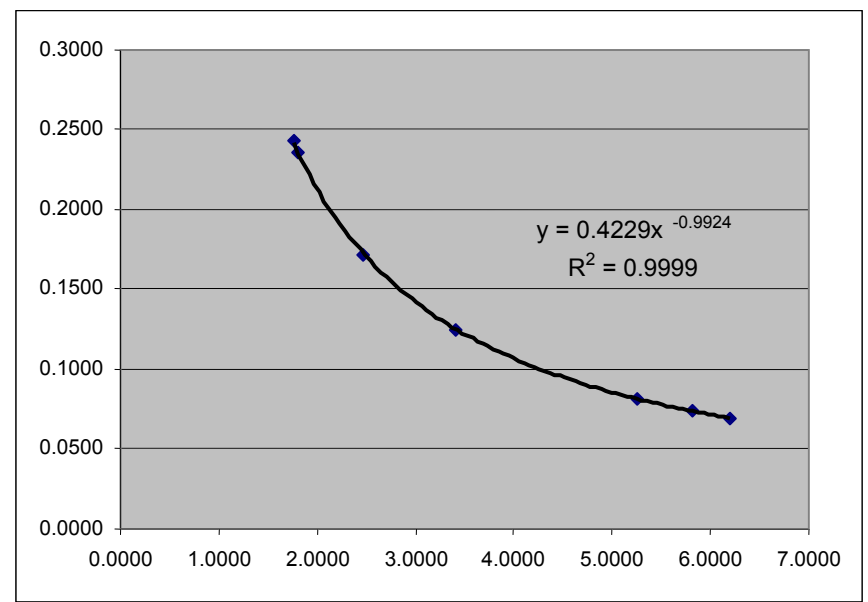

Figure S31. $f_{A}$ values calculated with the second model (Eq. 24) vs. $\eta_{A}$ parameter calculated with the first model (Eq. 8) for the molecule $\mathrm{CH}_{2} \mathrm{CHCN}$. The values of the two parameters have been obtained using the Hirshfeld population analysis.

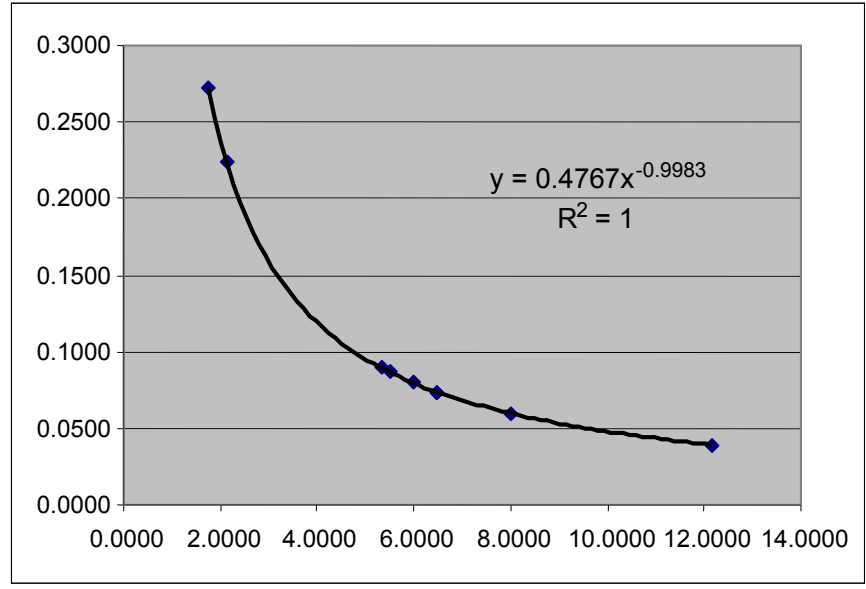

Figure S32. $f_{A}$ values calculated with the second model (Eq. 24) vs. $\eta_{A}$ parameter calculated with the first model (Eq. 8) for the molecule $\mathrm{CH}_{2} \mathrm{CHCH}_{3}$. The values of the two parameters have been obtained using the Hirshfeld population analysis. 


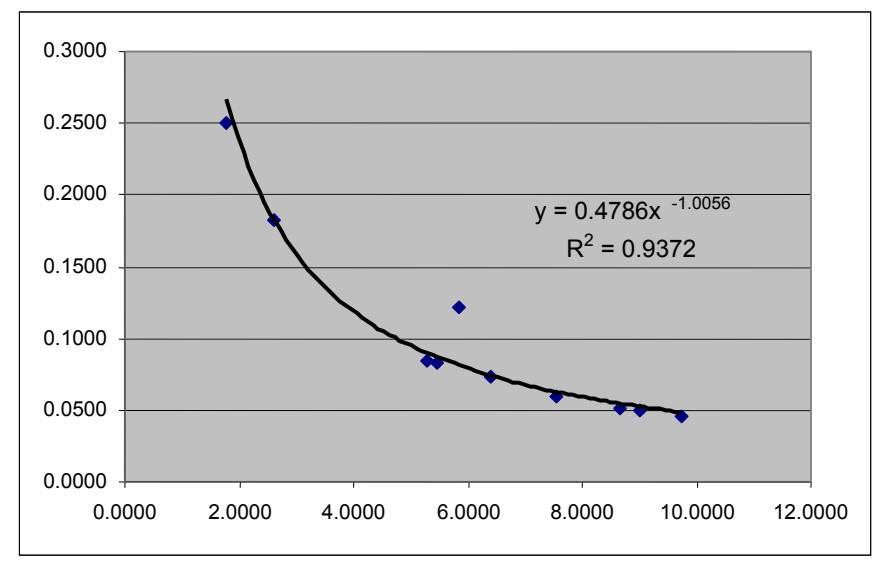

Figure S33. $f_{A}$ values calculated with the second model (Eq. 24) vs. $\eta_{A}$ parameter calculated with the first model (Eq. 8) for the molecule $\mathrm{CH}_{2} \mathrm{CHOCH}_{3}$. The values of the two parameters have been obtained using the Hirshfeld population analysis.

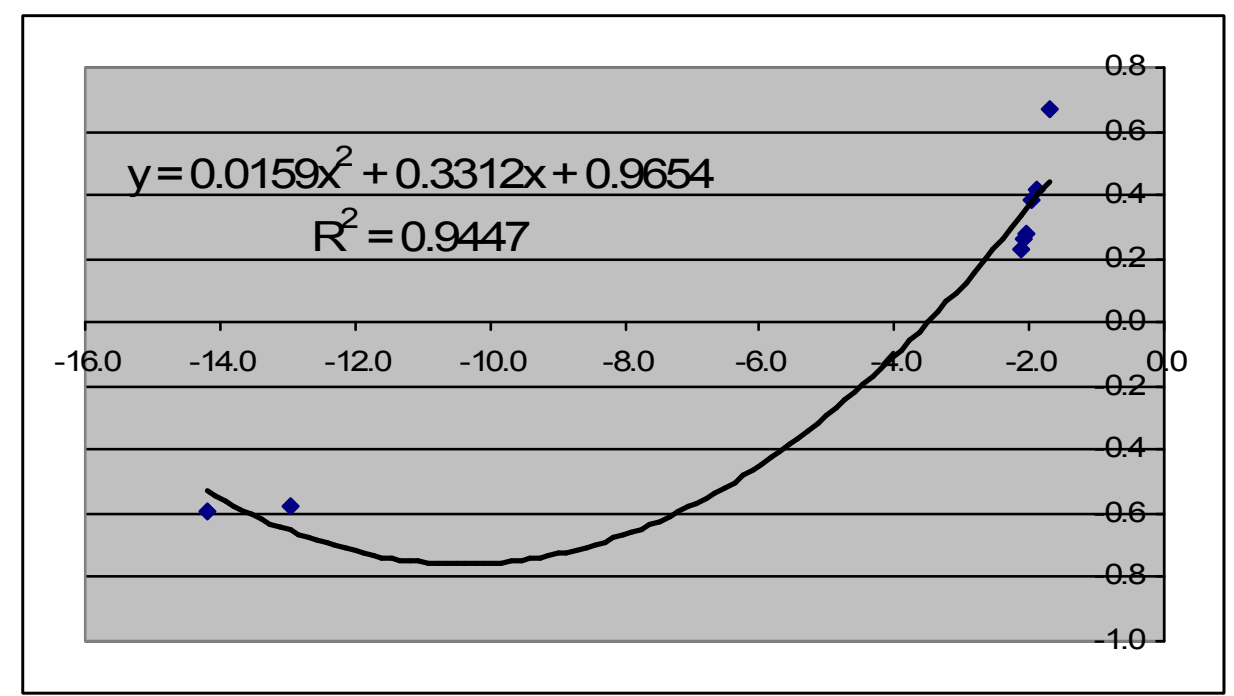

Figure S34. Parabolic regression $\left(f_{A}^{(2)} / f_{A}\right)$ versus $1 / \Omega_{A}$ for the molecule $\mathrm{CH}_{2} \mathrm{CHCHO}$. The values of the parameters have been obtained using the Hirshfeld population analysis. 


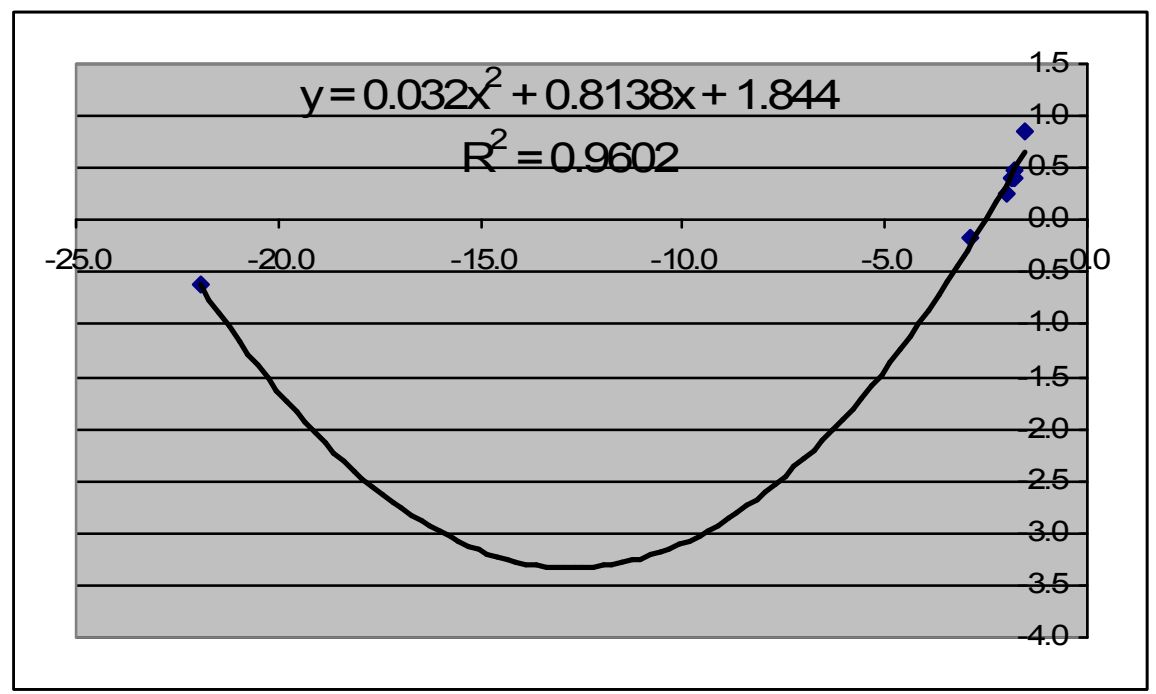

Figure S35. Parabolic regression $\left(f_{A}^{(2)} / f_{A}\right)$ versus $1 / \Omega_{A}$ for the molecule $\mathrm{CH}_{2} \mathrm{CHNO}_{2}$. The values of the parameters have been obtained using the Hirshfeld population analysis.

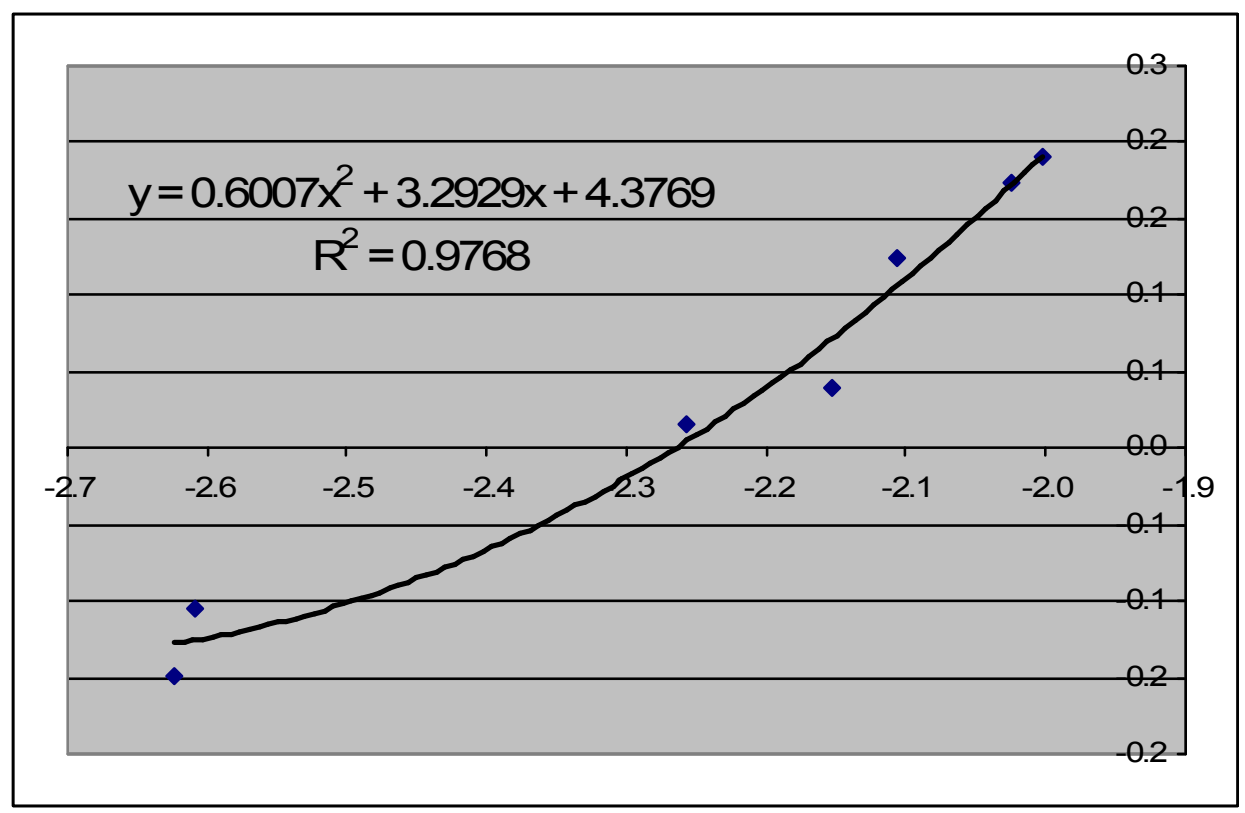

Figure S36. Parabolic regression $\left(f_{A}^{(2)} / f_{A}\right)$ versus $1 / \Omega_{A}$ for the molecule $\mathrm{CH}_{2} \mathrm{CHCN}$. The values of the parameters have been obtained using the Hirshfeld population analysis. 


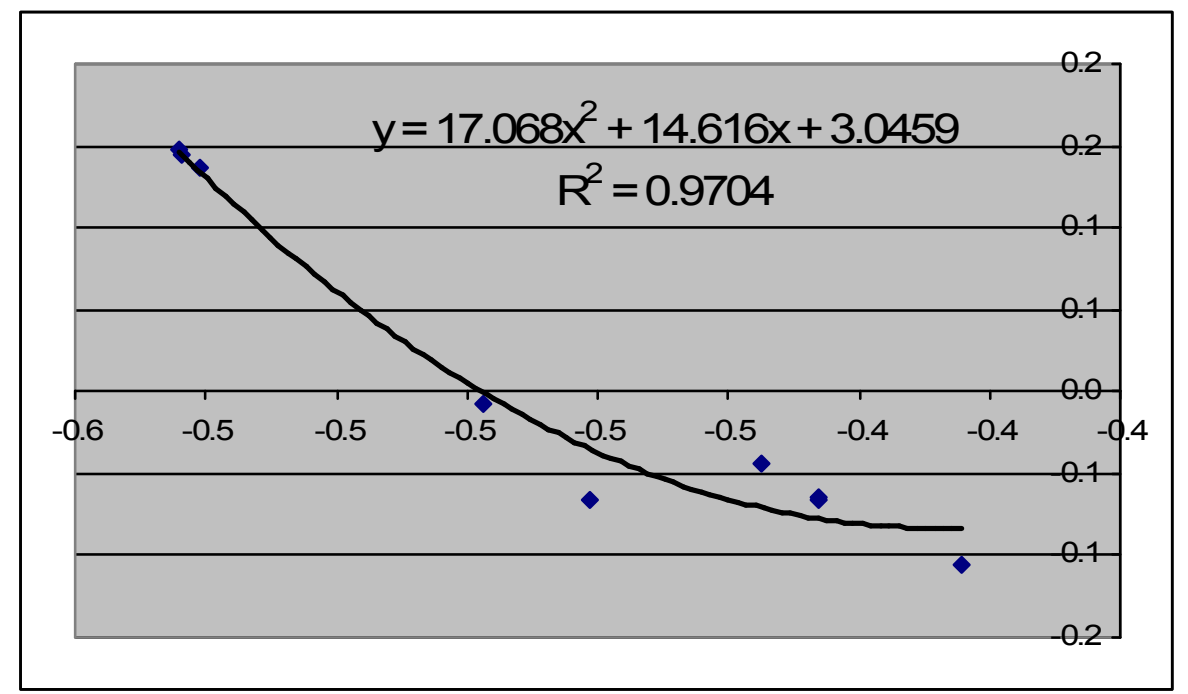

Figure S37. Parabolic regression $\left(f_{A}^{(2)} / f_{A}\right)$ versus $1 / \Omega_{A}$ for the molecule $\mathrm{CH}_{2} \mathrm{CHCH}_{3}$. The values of the parameters have been obtained using the Hirshfeld population analysis.

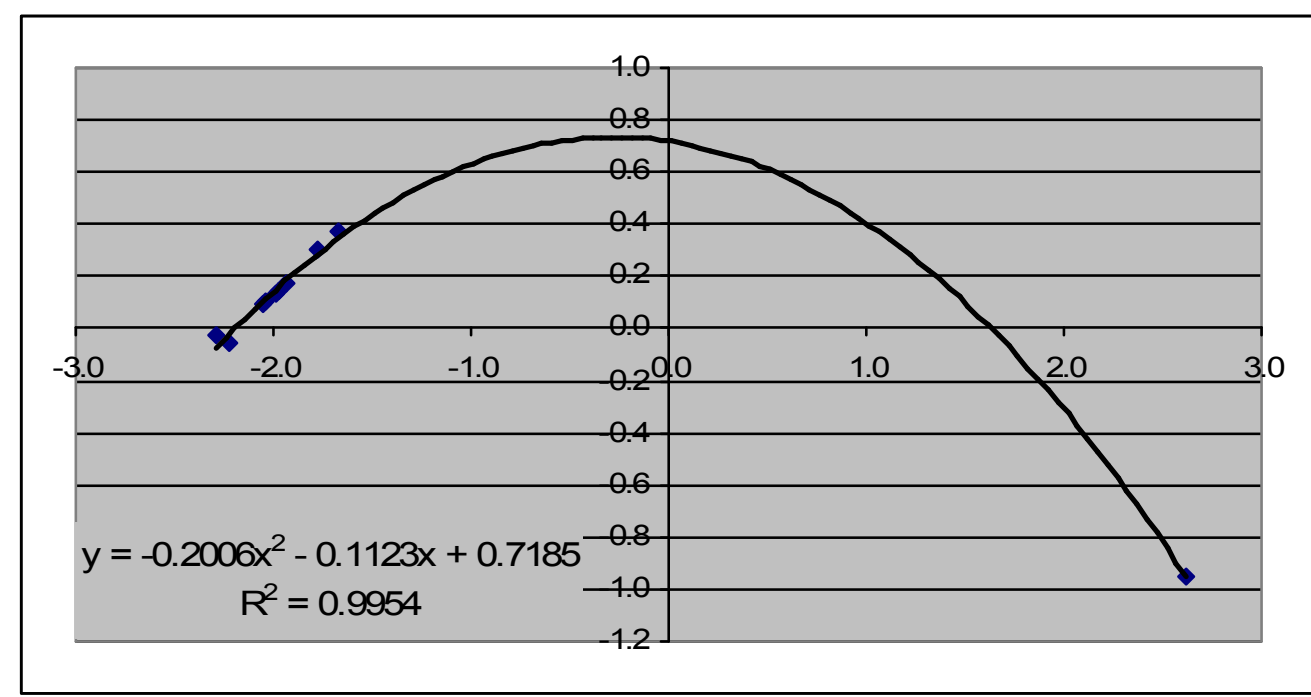

Figure S38. Parabolic regression $\left(f_{A}^{(2)} / f_{A}\right)$ versus $1 / \Omega_{A}$ for the molecule $\mathrm{CH}_{2} \mathrm{CHOCH}_{3}$.

The values of the parameters have been obtained using the Hirshfeld population analysis. 


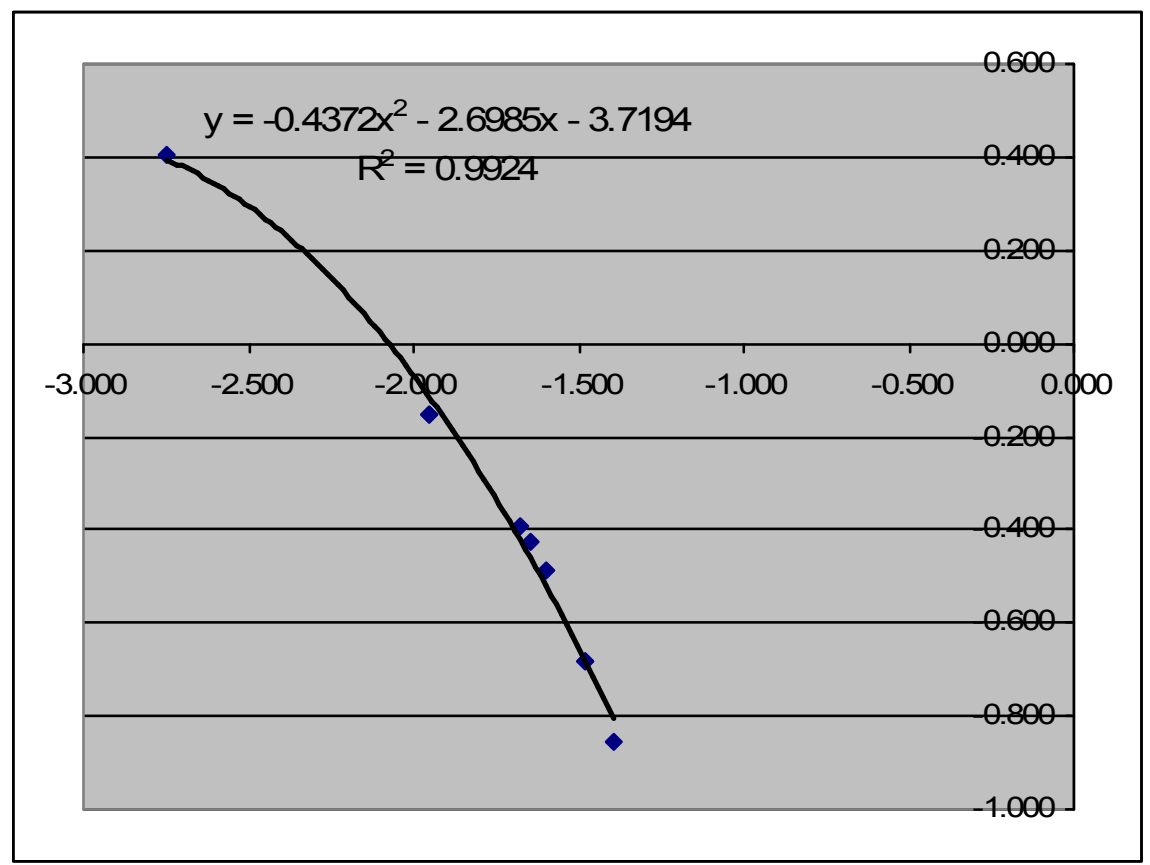

Figure S39. Parabolic regression $\left(f_{A}^{(2)} / f_{A}\right)$ versus $1 / \Omega_{A}$ for the molecule $\mathrm{CH}_{3} \mathrm{CHSH}$. The values of the parameters have been obtained using the Hirshfeld population analysis.

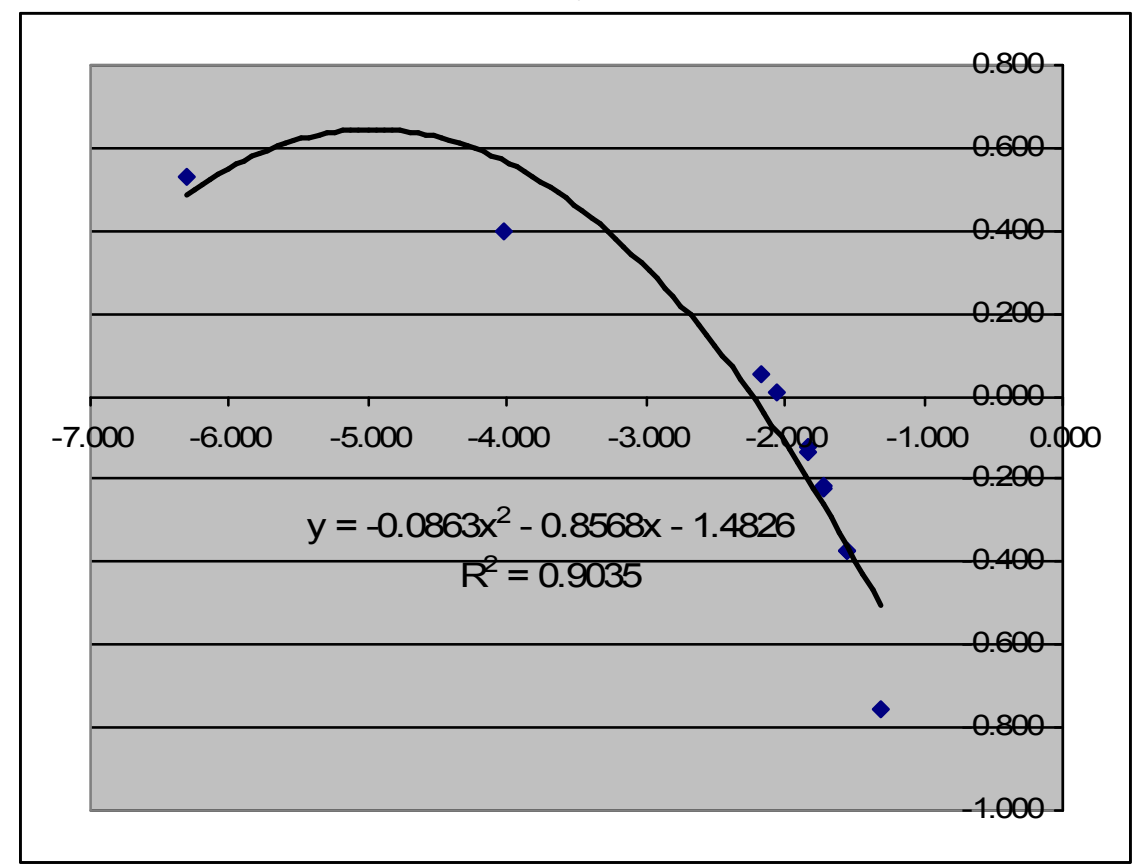

Figure S40. Parabolic regression $\left(f_{A}^{(2)} / f_{A}\right)$ versus $1 / \Omega_{A}$ for the molecule $\mathrm{CH}_{3} \mathrm{COOCH}_{3}$. The values of the parameters have been obtained using the Hirshfeld population analysis. 


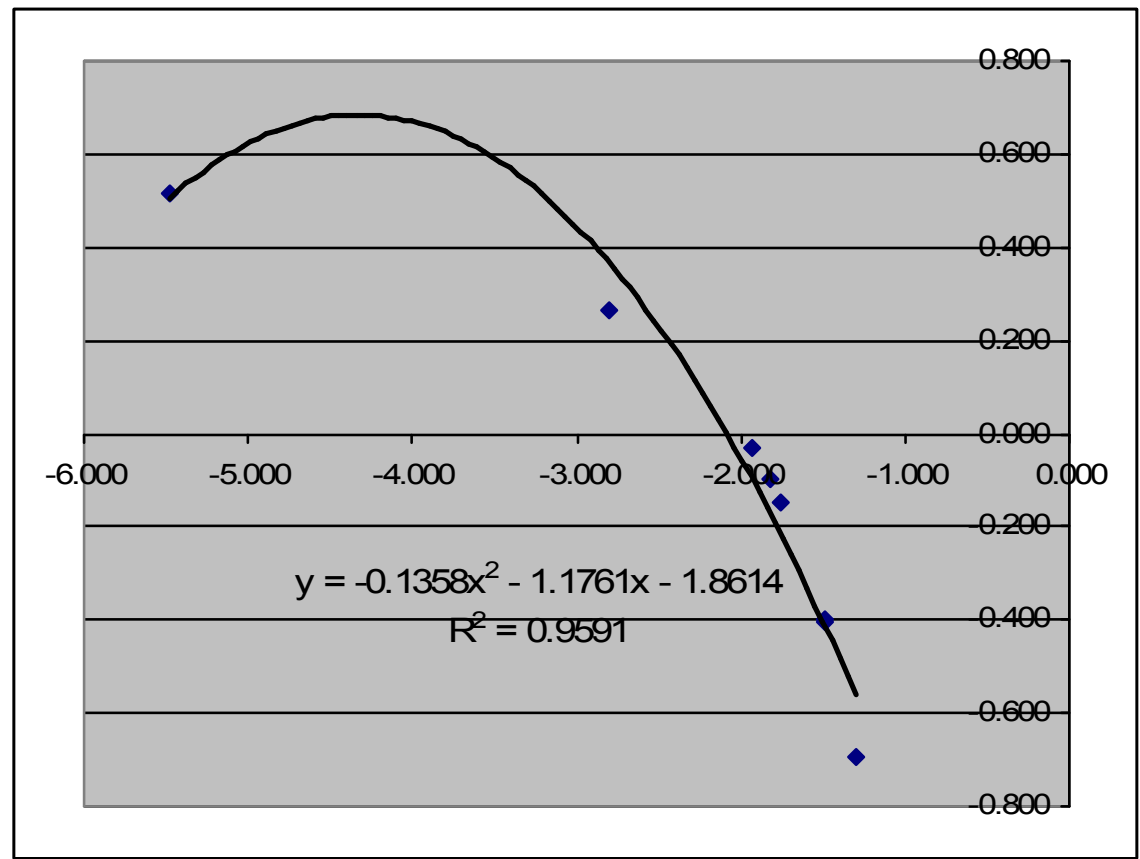

Figure S41. Parabolic regression $\left(f_{A}^{(2)} / f_{A}\right)$ versus $1 / \Omega_{A}$ for the molecule $\mathrm{CH}_{3} \mathrm{COOH}$. The values of the parameters have been obtained using the Hirshfeld population analysis.

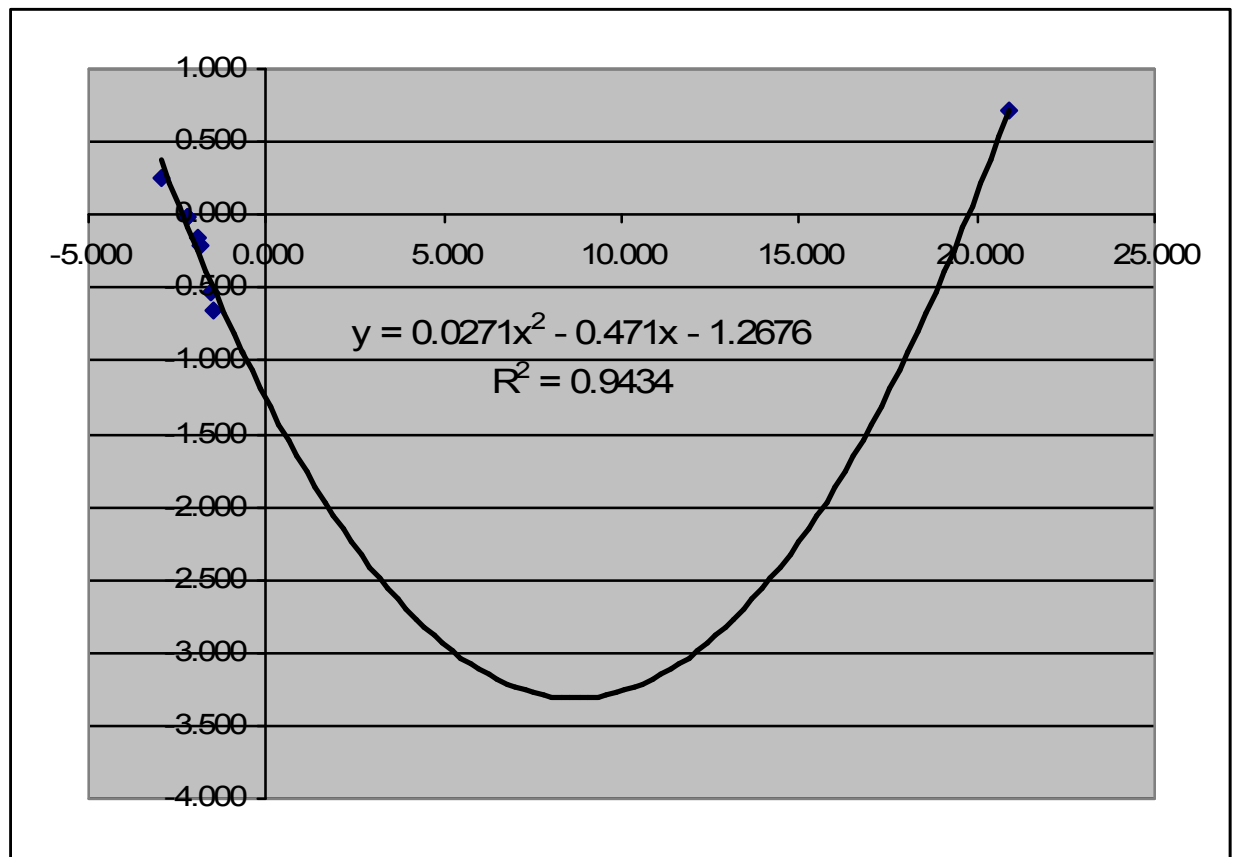

Figure S42. Parabolic regression $\left(f_{A}^{(2)} / f_{A}\right)$ versus $1 / \Omega_{A}$ for the molecule $\mathrm{CH}_{2} \mathrm{CHNH}_{2}$. The values of the parameters have been obtained using the Hirshfeld population analysis. 


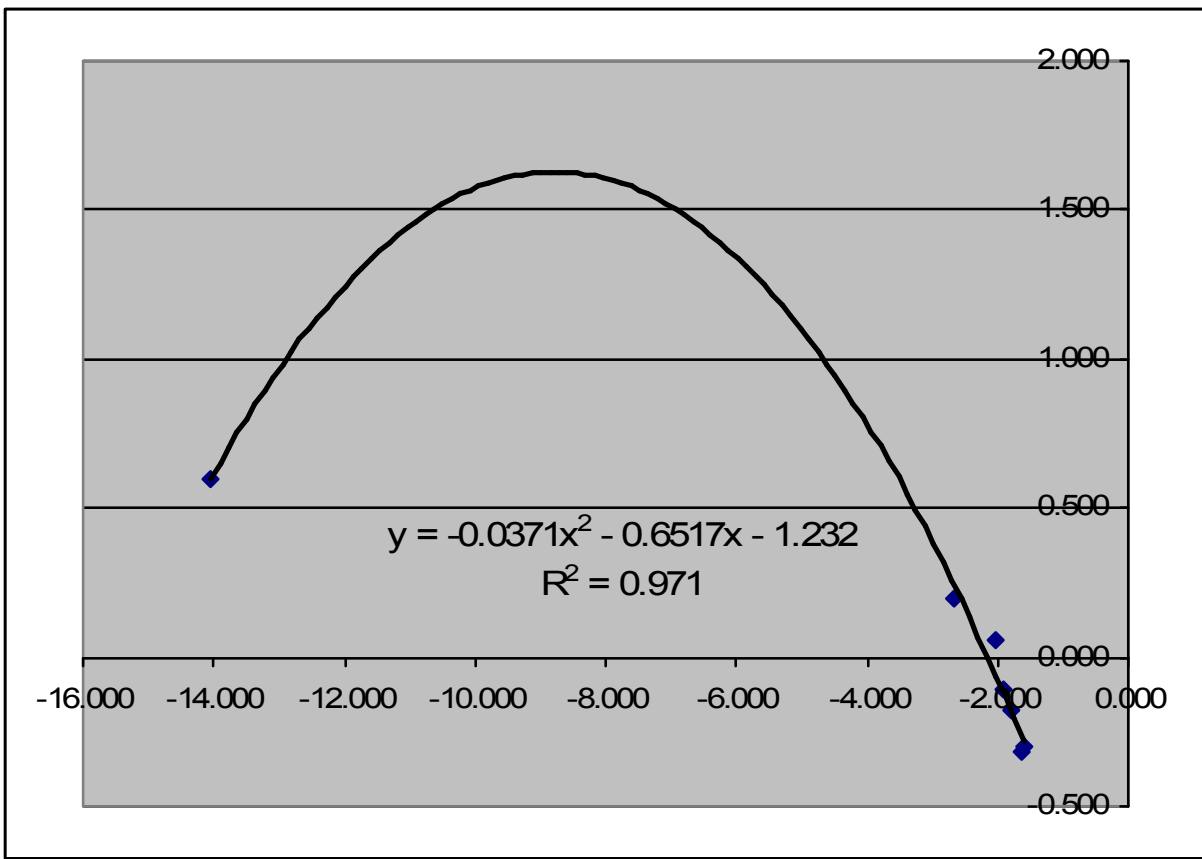

Figure S43. Parabolic regression $\left(f_{A}^{(2)} / f_{A}\right)$ versus $1 / \Omega_{A}$ for the molecule $\mathrm{CH}_{2} \mathrm{CHOH}$. The values of the parameters have been obtained using the Hirshfeld population analysis.

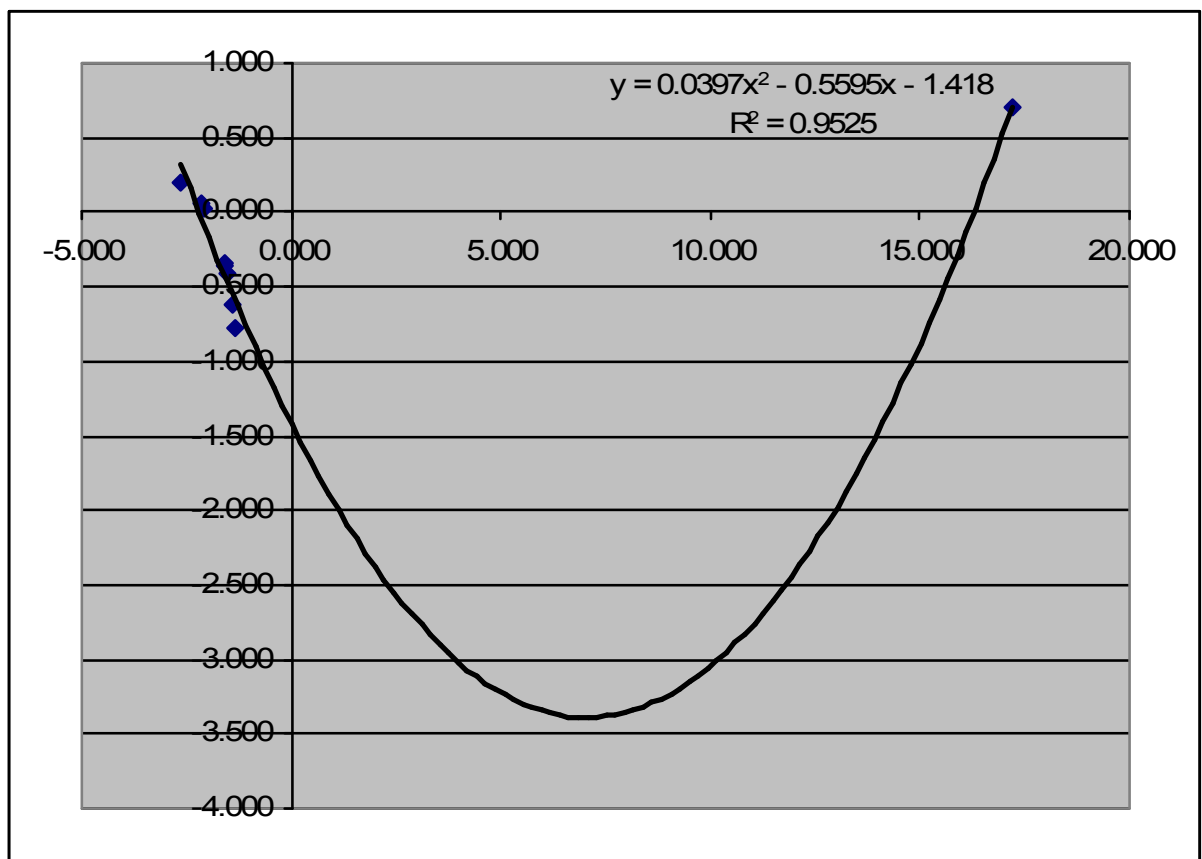

Figure S44. Parabolic regression $\left(f_{A}^{(2)} / f_{A}\right)$ versus $1 / \Omega_{A}$ for the molecule $\mathrm{CH}_{3} \mathrm{CONH}_{2}$. The values of the parameters have been obtained using the Hirshfeld population analysis. 


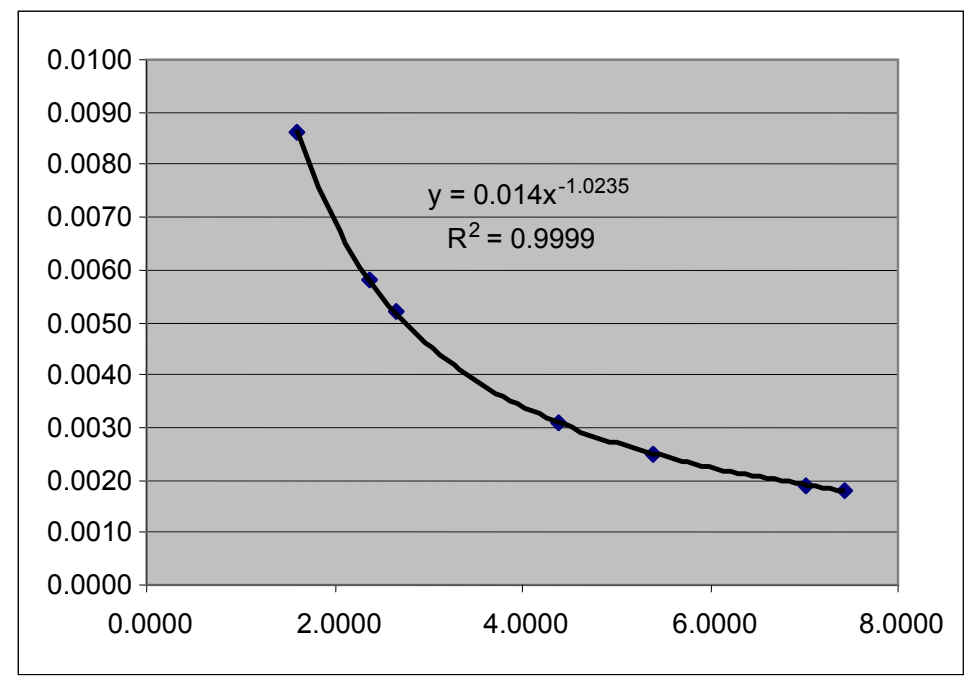

Figure S45. Electrophilicities $\omega_{A}$ versus the local parameter $\eta_{A}$ for the $\mathrm{CH}_{2} \mathrm{CHCHO}$ molecule. We have used the Hirshfeld population analysis in both cases.

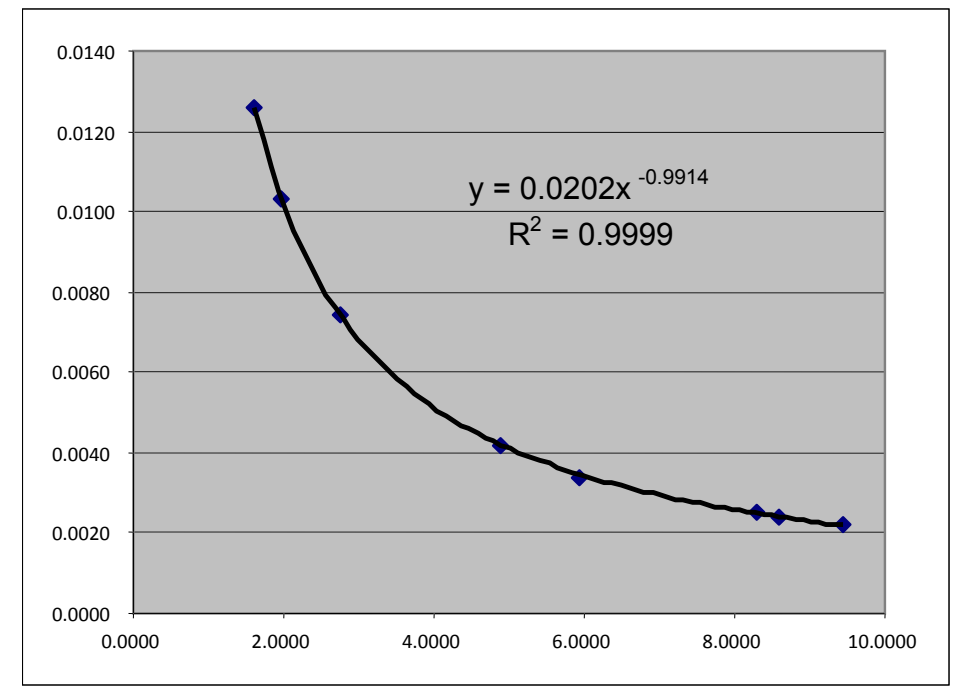

Figure S46. Electrophilicities $\omega_{A}$ versus the local parameter $\eta_{A}$ for the $\mathrm{CH}_{2} \mathrm{CHNO}_{2}$ molecule. We have used the Hirshfeld population analysis in both cases. 


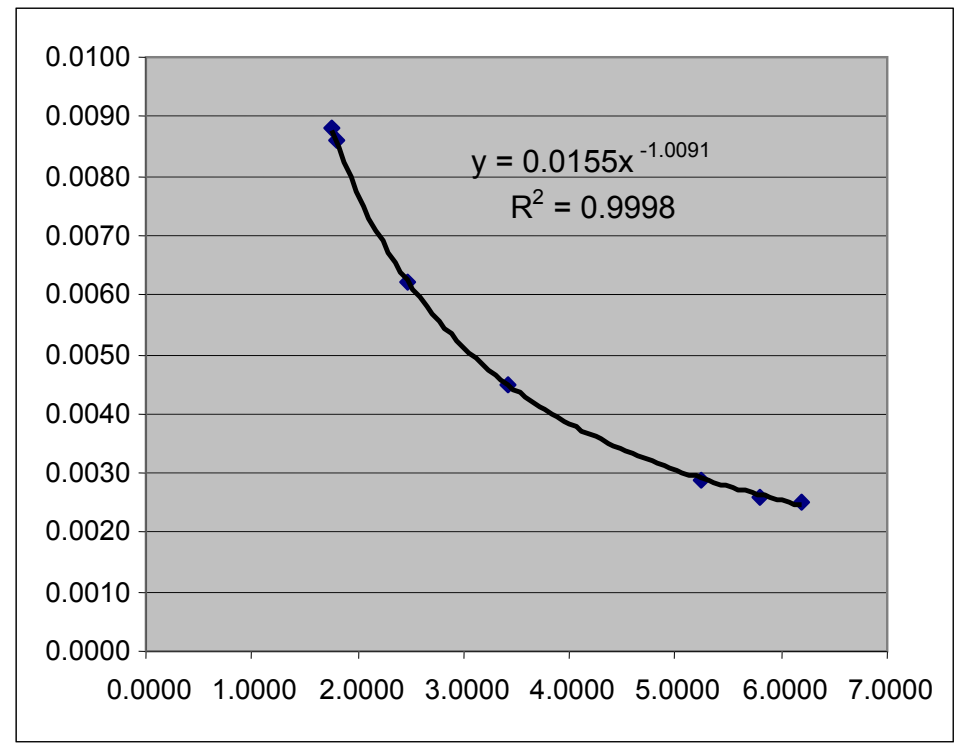

Figure S47. Electrophilicities $\omega_{A}$ versus the local parameter $\eta_{A}$ for the $\mathrm{CH}_{2} \mathrm{CHCN}$ molecule. We have used the Hirshfeld population analysis in both cases.

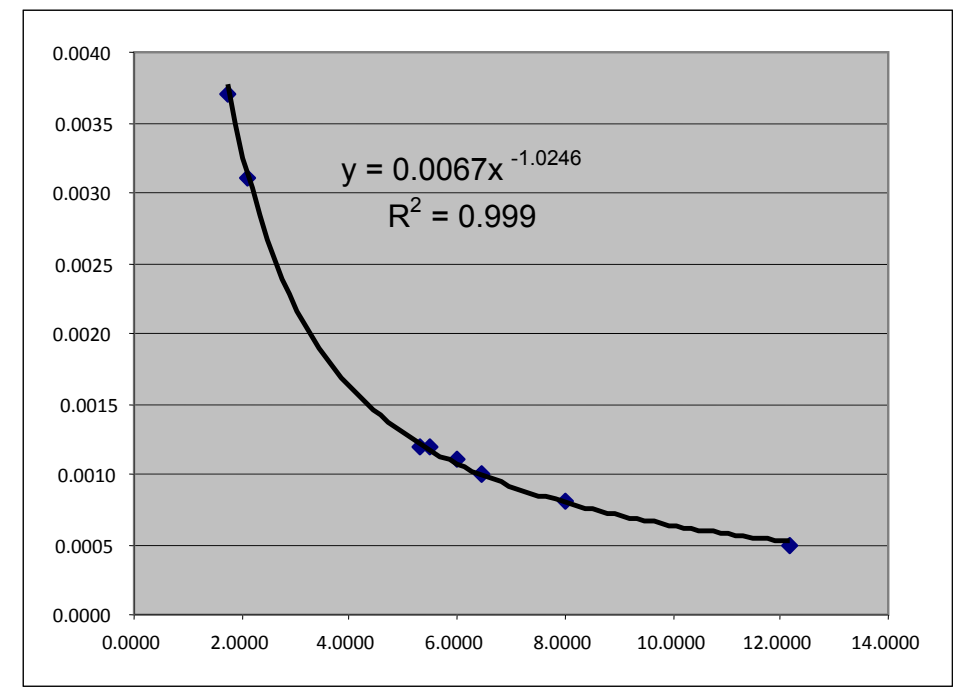

Figure S48. Electrophilicities $\omega_{A}$ versus the local parameter $\eta_{A}$ for the $\mathrm{CH}_{2} \mathrm{CHCH}_{3}$ molecule. We have used the Hirshfeld population analysis in both cases. 


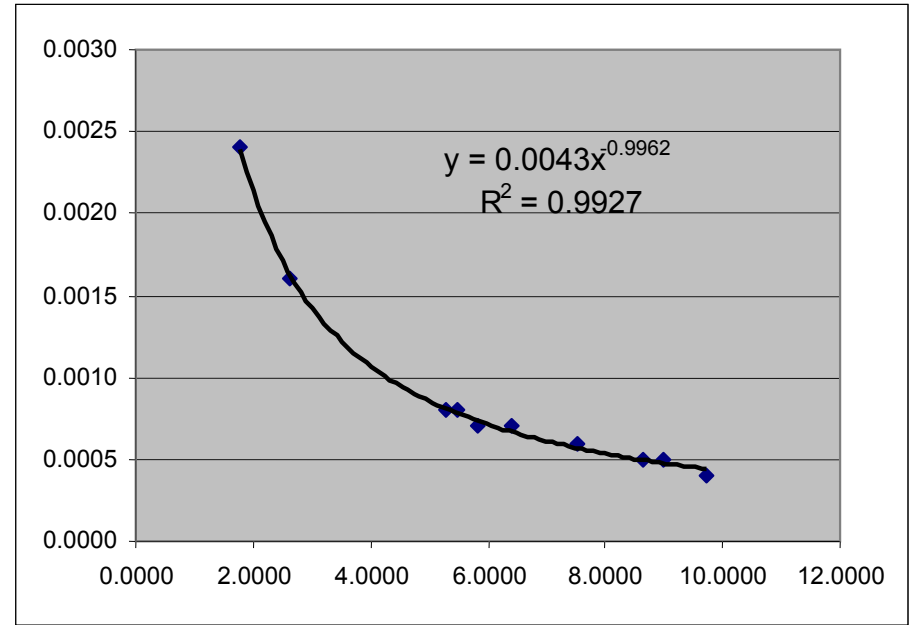

Figure S49. Electrophilicities $\omega_{A}$ versus the local parameter $\eta_{A}$ for the $\mathrm{CH}_{2} \mathrm{CHOCH}_{3}$ molecule. We have used the Hirshfeld population analysis in both cases. 


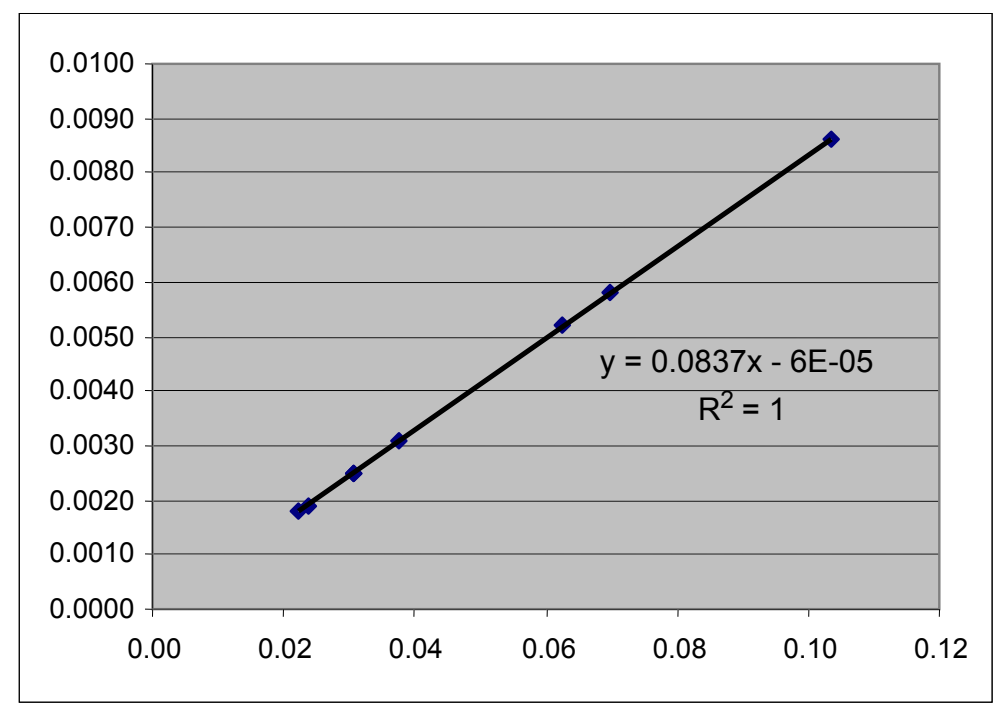

Figure S50. Atomic values $\omega_{A}$ versus local parameter $\Delta N_{A}^{\max }$ for the $\mathrm{CH}_{2} \mathrm{CHCHO}$ molecule using the Hirshfeld population analysis.

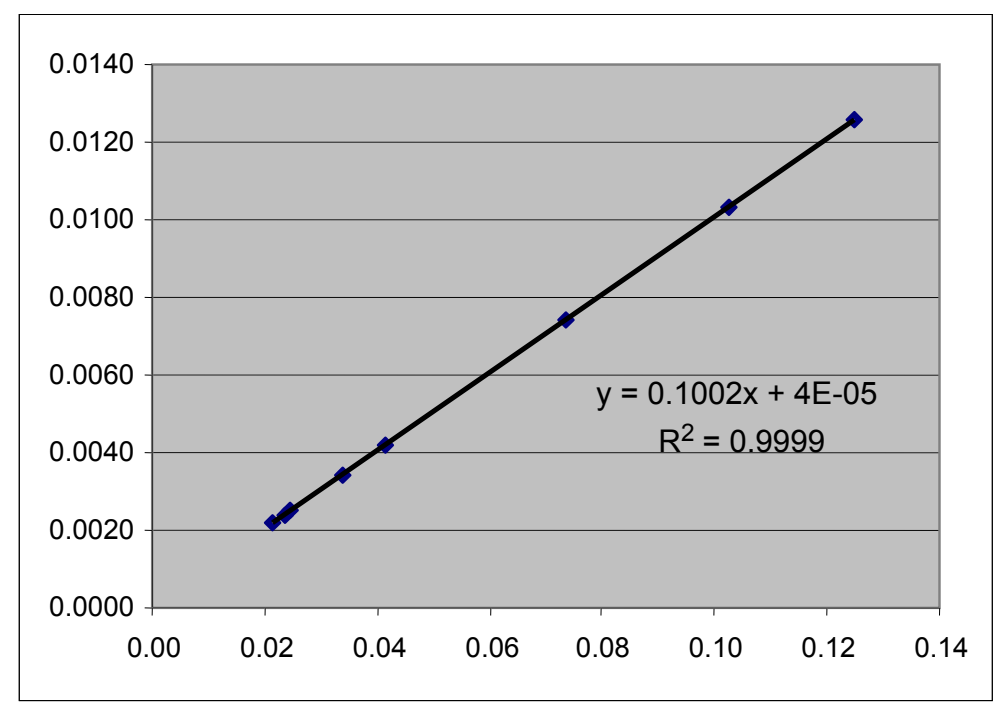

Figure S51. Atomic values $\omega_{A}$ versus local parameter $\Delta N_{A}^{\max }$ for the $\mathrm{CH}_{2} \mathrm{CHNO}_{2}$ molecule using the Hirshfeld population analysis. 


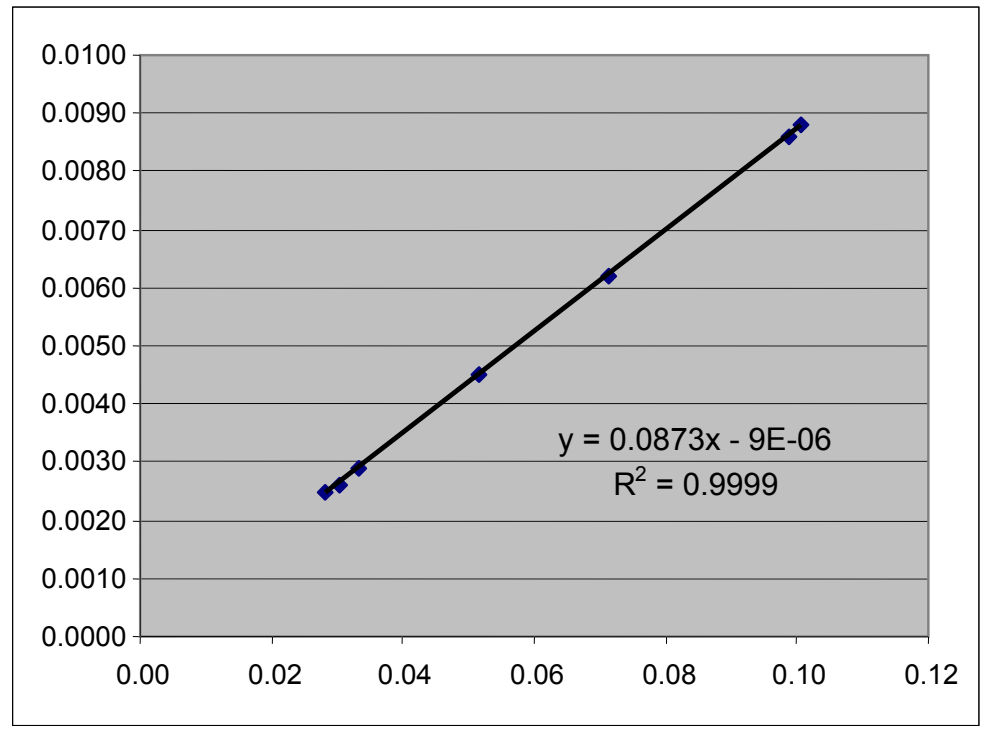

Figure S52. Atomic values $\omega_{A}$ versus local parameter $\Delta N_{A}^{\max }$ for the $\mathrm{CH}_{2} \mathrm{CHCN}$ molecule using the Hirshfeld population analysis.

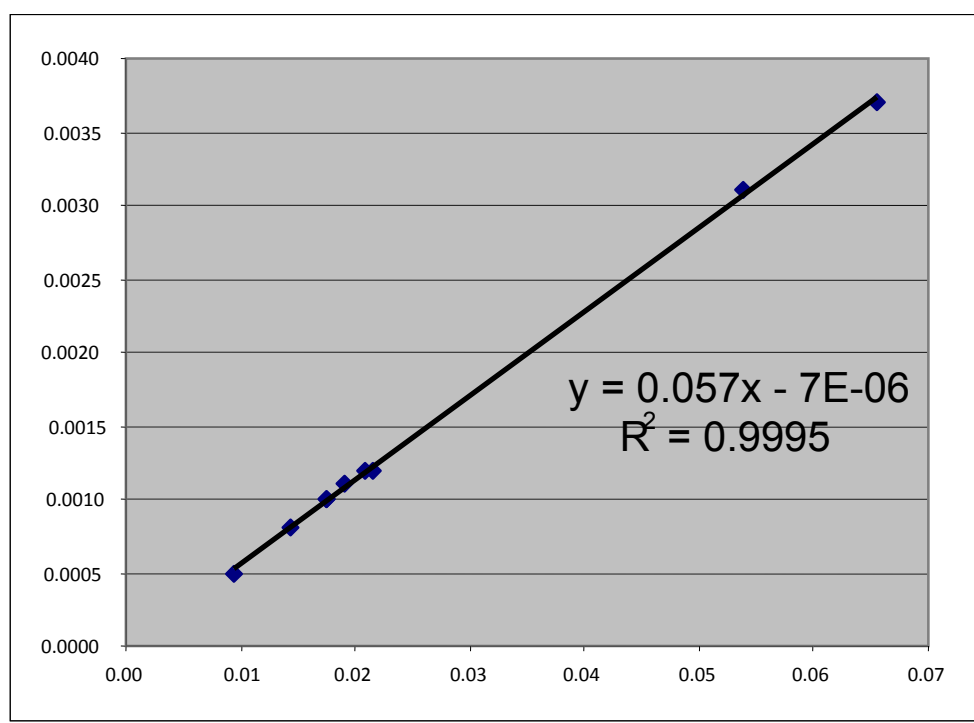

Figure S53. Atomic values $\omega_{A}$ versus local parameter $\Delta N_{A}^{\max }$ for the $\mathrm{CH}_{2} \mathrm{CHCH}_{3}$ molecule using the Hirshfeld population analysis. 


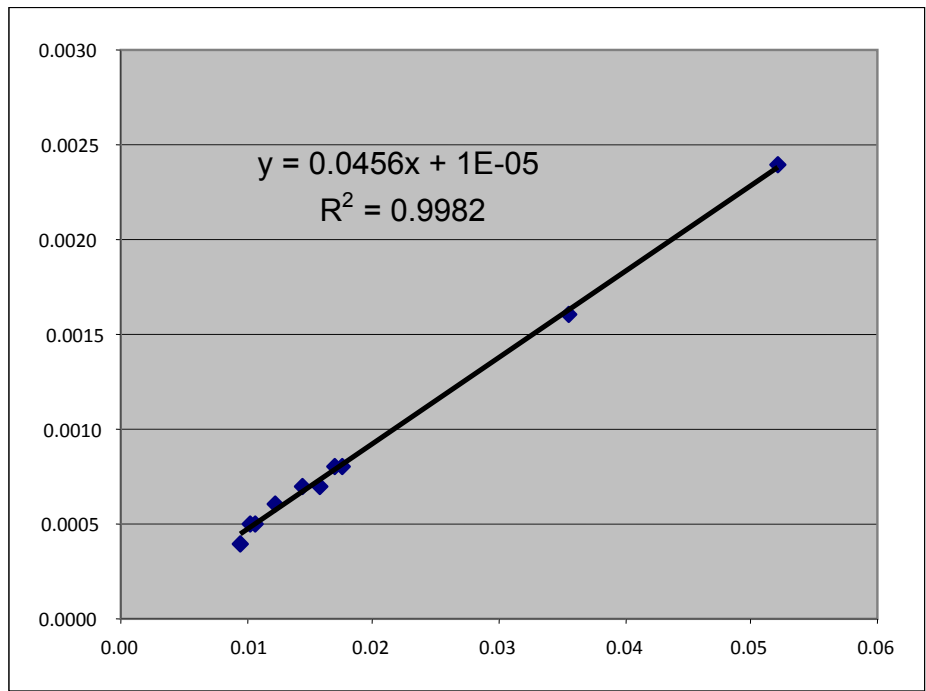

Figure S54. Atomic values $\omega_{A}$ versus local parameter $\Delta N_{A}^{\max }$ for the $\mathrm{CH}_{2} \mathrm{CHOCH}_{3}$ molecule using the Hirshfeld population analysis. 


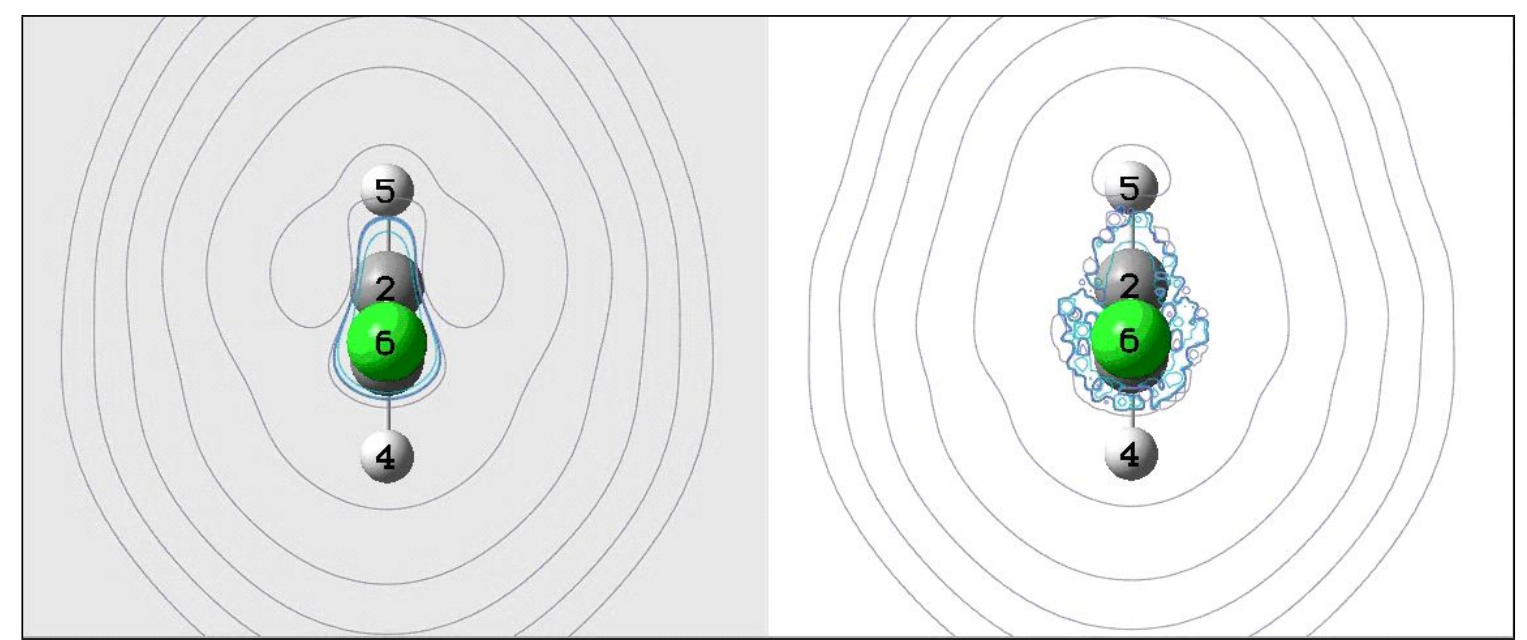

Figure S55. Left) Fukui function $f(\mathbf{r})_{\text {Quadratic expansion }}$ calculated with a parabolic approximation (Eq. 23) and right) hardness function $\eta(\mathbf{r})$ (Eq. 13) for the $\mathrm{CH}_{2} \mathrm{CHCl}$ molecule.

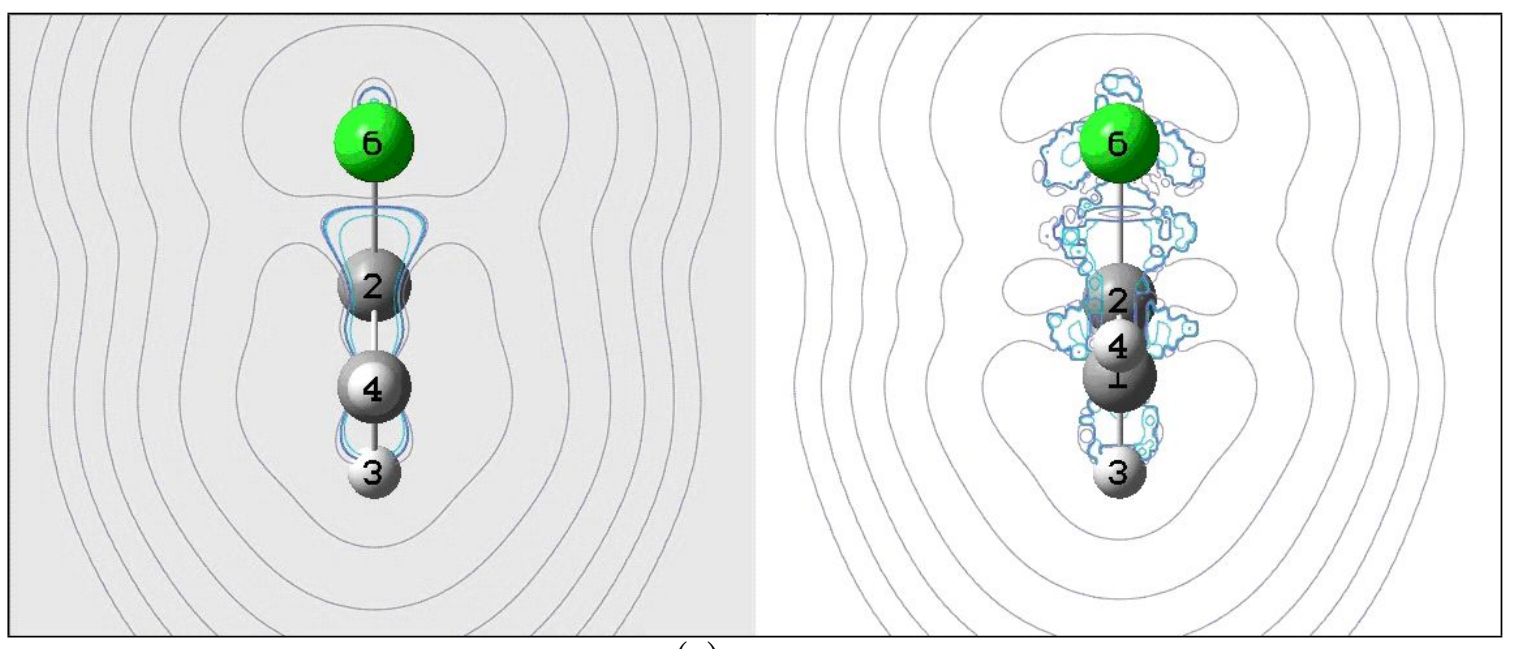

Figure S56. Left) Fukui function $f(\mathbf{r})_{\text {Quadratic expansion }}$ calculated with a parabolic approximation (Eq. 23) and right) hardness function $\eta(\mathbf{r})$ (Eq. 13) for the $\mathrm{CH}_{2} \mathrm{CHCl}$ molecule. 


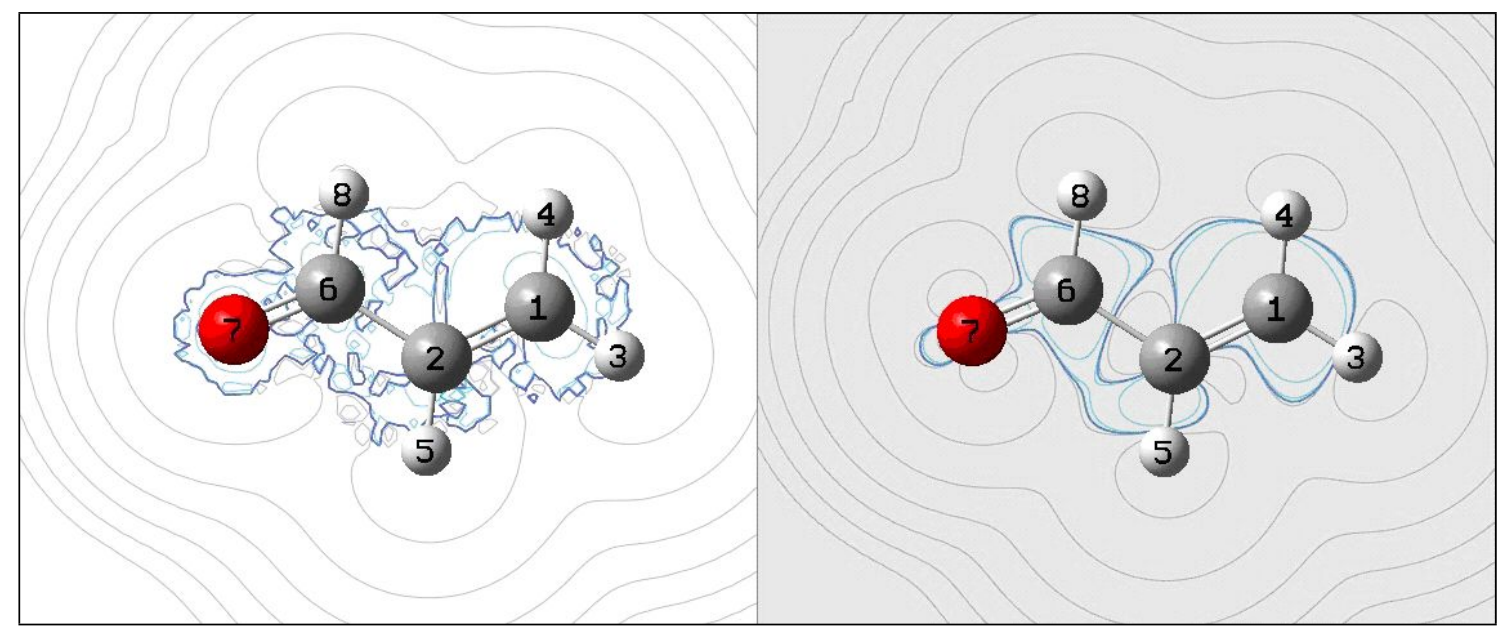

Figure S57. Left) Fukui function $f(\mathbf{r})_{\text {Quadratic expansion }}$ calculated with a parabolic approximation (Eq. 23) and right) hardness function $\eta(\mathbf{r})$ (Eq. 13) for the $\mathrm{CH}_{2} \mathrm{CHCHO}$ molecule.

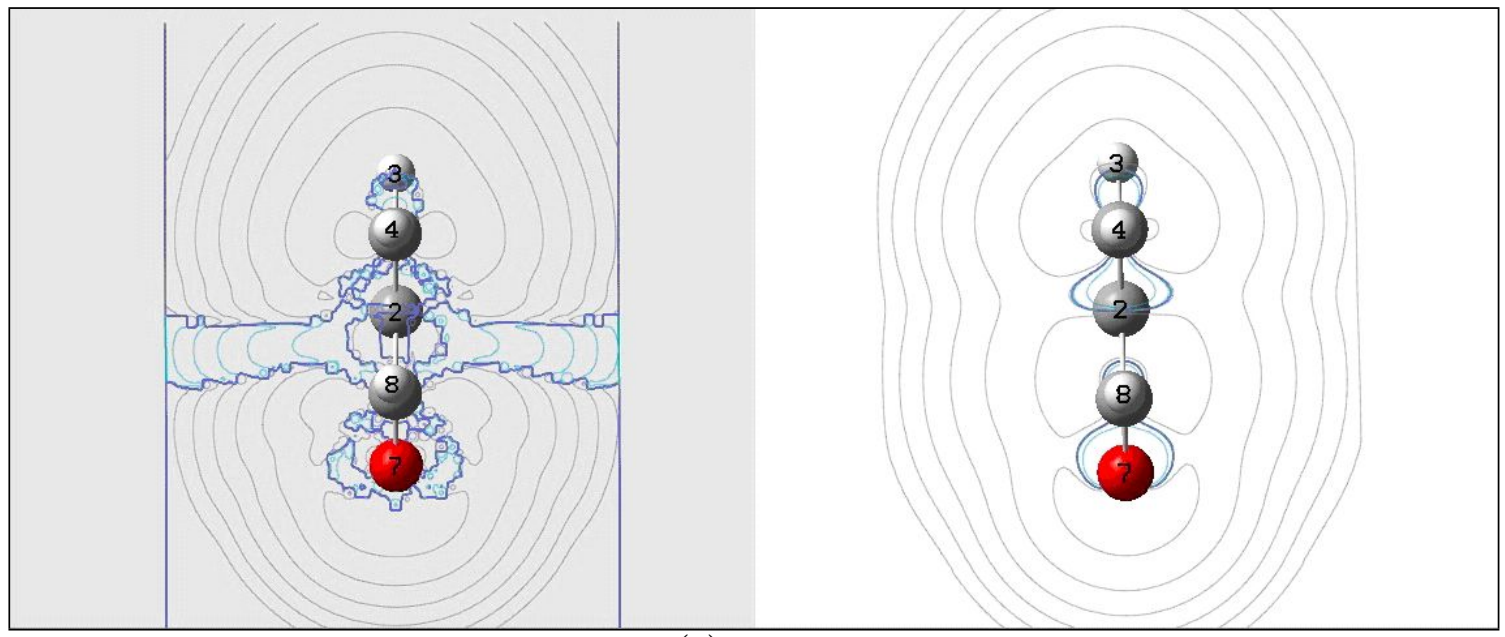

Figure S58. Left) Fukui function $f(\mathbf{r})_{\text {Quadratic expansion }}$ calculated with a parabolic approximation (Eq. 23) and right) hardness function $\eta(\mathbf{r})$ (Eq. 13) for the $\mathrm{CH}_{2} \mathrm{CHCHO}$ molecule. 


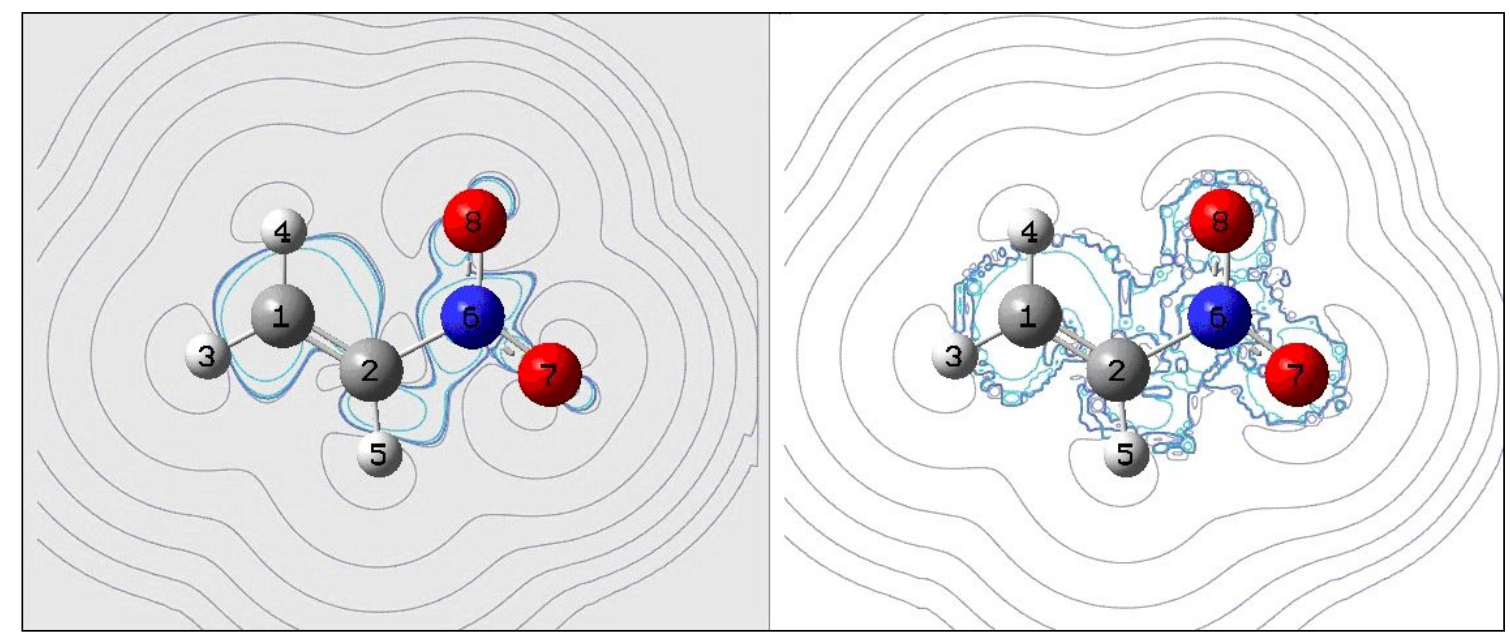

Figure S59. Left) Fukui function $f(\mathbf{r})_{\text {Quadratic expansion }}$ calculated with a parabolic approximation (Eq. 23) and right) hardness function $\eta(\mathbf{r})$ (Eq. 13) for the $\mathrm{CH}_{2} \mathrm{CHNO}_{2}$ molecule.

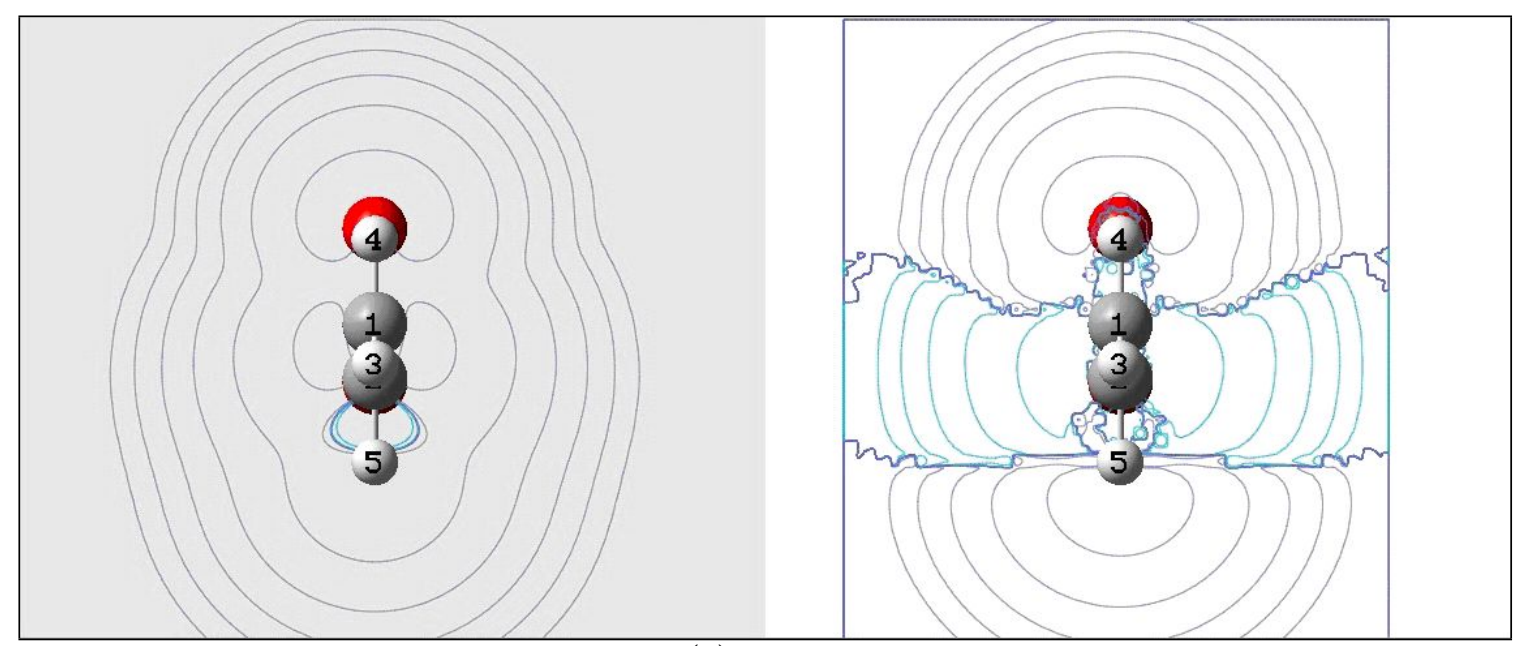

Figure S60. Left) Fukui function $f(\mathbf{r})_{\text {Quadratic expansion }}$ calculated with a parabolic approximation (Eq. 23) and right) hardness function $\eta(\mathbf{r})$ (Eq. 13) for the $\mathrm{CH}_{2} \mathrm{CHNO}_{2}$ molecule. 


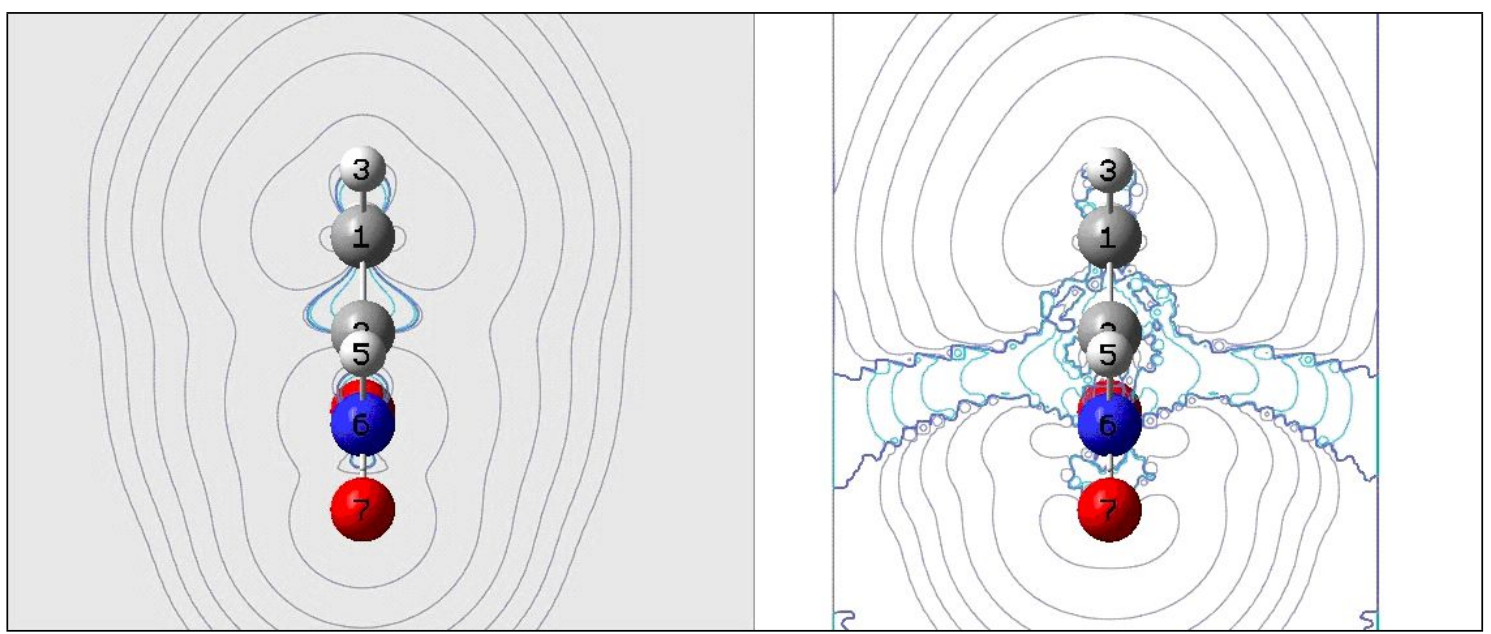

Figure S61. Left) Fukui function $f(\mathbf{r})_{\text {Quadratic expansion }}$ calculated with a parabolic approximation (Eq. 23) and right) hardness function $\eta(\mathbf{r})$ (Eq. 13) for the $\mathrm{CH}_{2} \mathrm{CHNO}_{2}$ molecule.

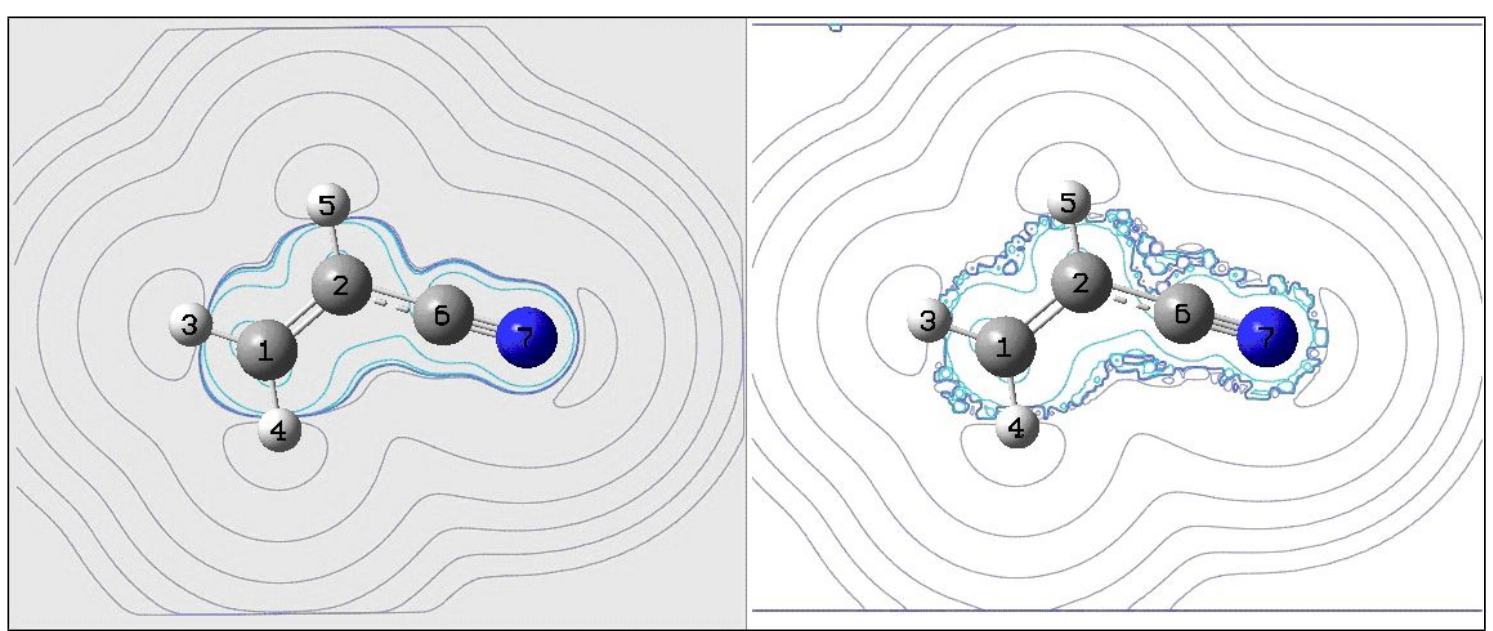

Figure S62. Left) Fukui function $f(\mathbf{r})_{\text {Quadratic expansion }}$ calculated with a parabolic approximation (Eq. 23) and right) hardness function $\eta(\mathbf{r})$ (Eq. 13) for the $\mathrm{CH}_{2} \mathrm{CHCN}$ molecule. 


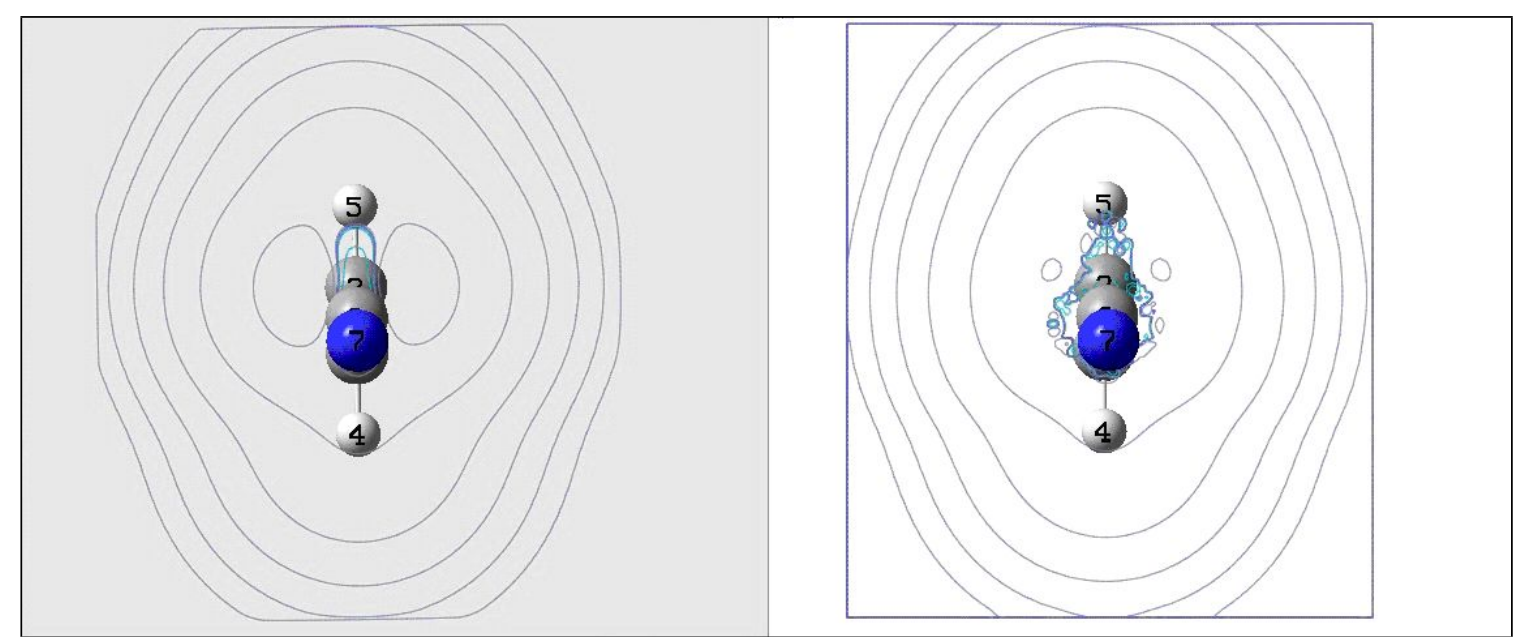

Figure S63. Left) Fukui function $f(\mathbf{r})_{\text {Quadratic expansion }}$ calculated with a parabolic approximation (Eq. 23) and right) hardness function $\eta(\mathbf{r})$ (Eq. 13) for the $\mathrm{CH}_{2} \mathrm{CHCN}$ molecule.

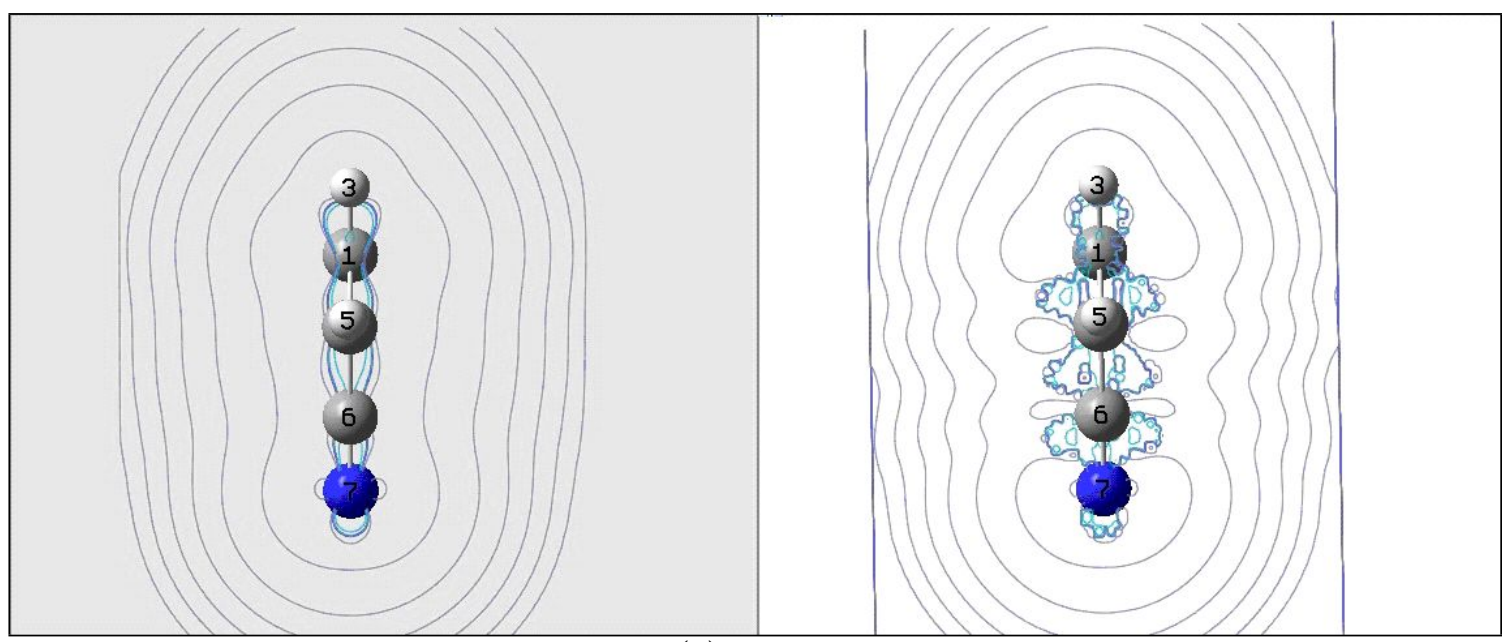

Figure S64. Left) Fukui function $f(\mathbf{r})_{\text {Quadratic expansion }}$ calculated with a parabolic approximation (Eq. 23) and right) hardness function $\eta(\mathbf{r})$ (Eq. 13) for the $\mathrm{CH}_{2} \mathrm{CHCN}$ molecule. 


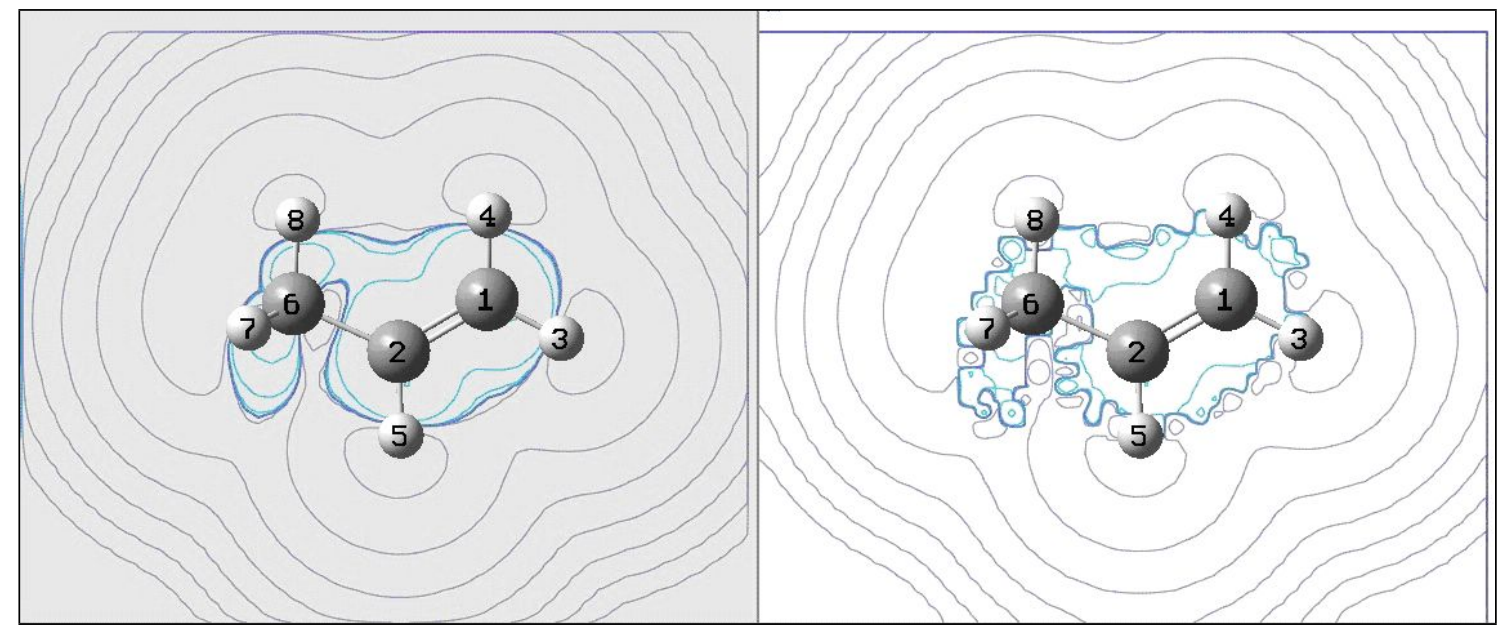

Figure S65. Left) Fukui function $f(\mathbf{r})_{\text {Quadratic expansion }}$ calculated with a parabolic approximation (Eq. 23) and right) hardness function $\eta(\mathbf{r})$ (Eq. 13) for the $\mathrm{CH}_{2} \mathrm{CHCH}_{3}$ molecule.

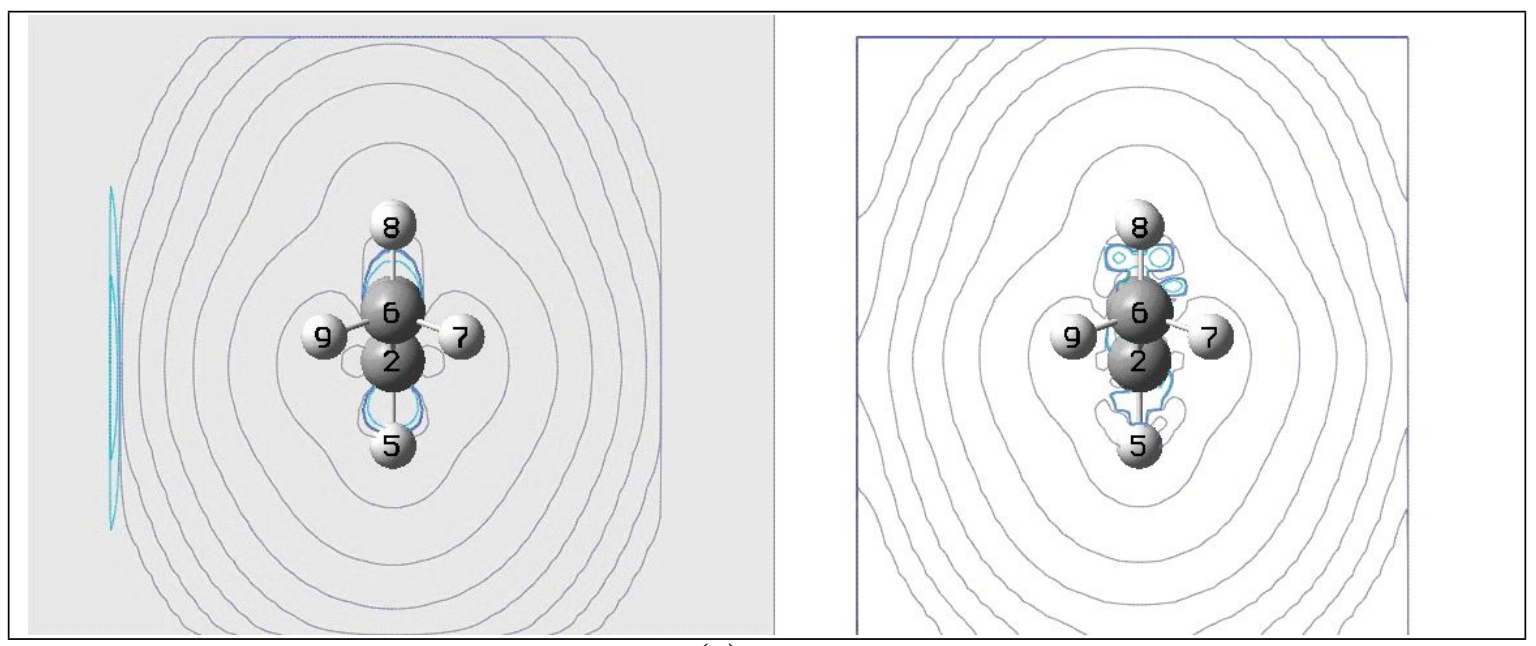

Figure S66. Left) Fukui function $f(\mathbf{r})_{\text {Quadratic expansion }}$ calculated with a parabolic approximation (Eq. 23) and right) hardness function $\eta(\mathbf{r})$ (Eq. 13) for the $\mathrm{CH}_{2} \mathrm{CHCH}_{3}$ molecule. 


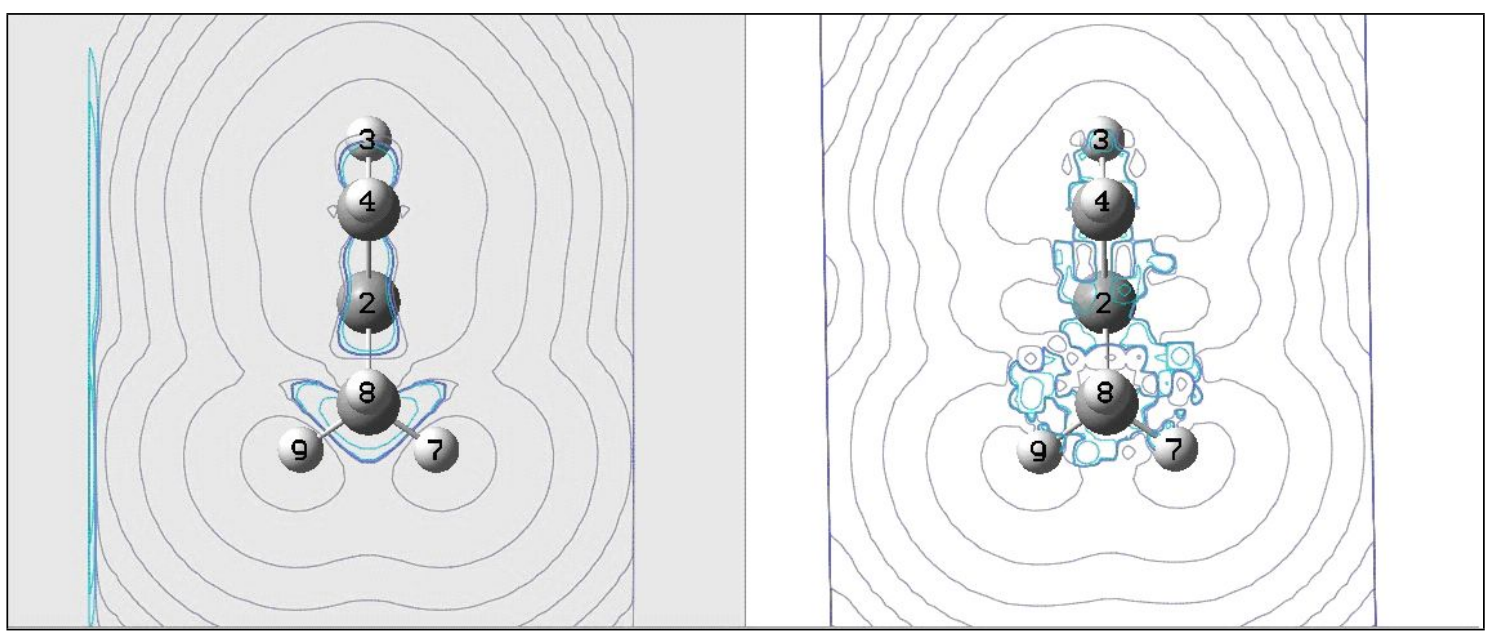

Figure S67. Left) Fukui function $f(\mathbf{r})_{\text {Quadratic expansion }}$ calculated with a parabolic approximation (Eq. 23) and right) hardness function $\eta(\mathbf{r})$ (Eq. 13) for the $\mathrm{CH}_{2} \mathrm{CHCH}_{3}$ molecule.

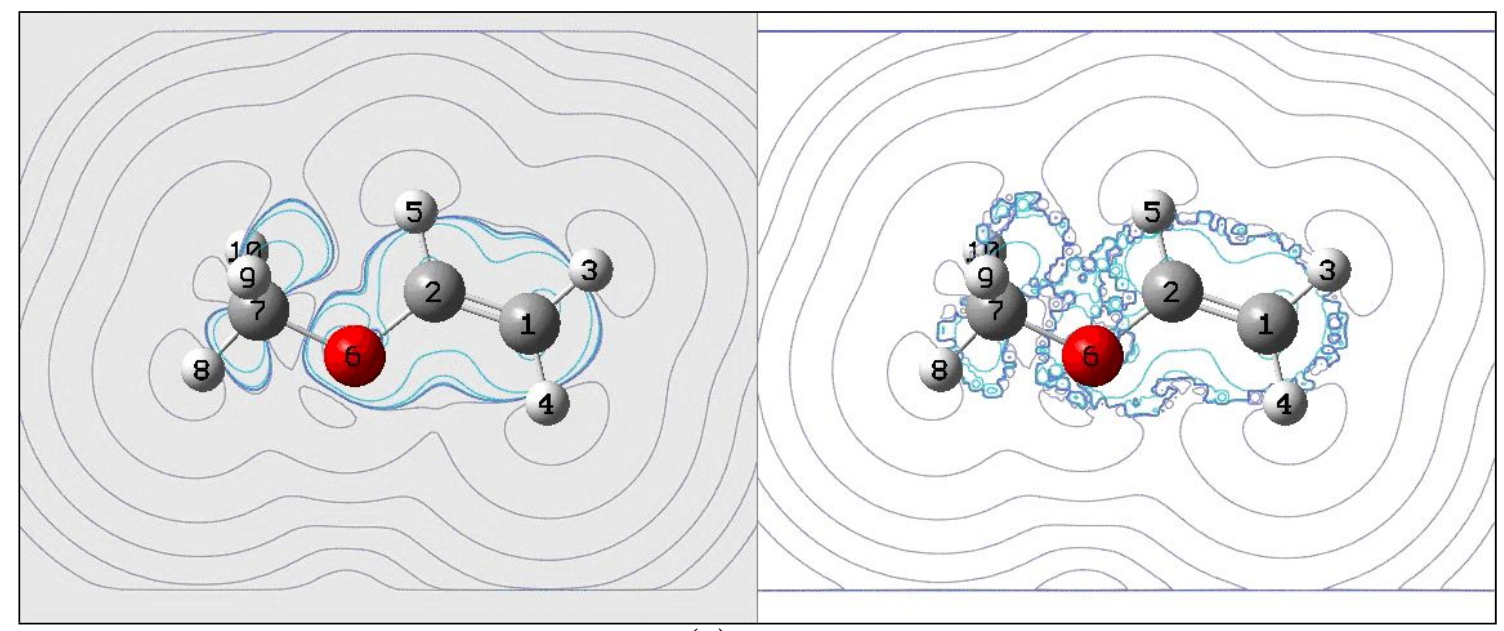

Figure S68. Left) Fukui function $f(\mathbf{r})_{\text {Quadratic expansion }}$ calculated with a parabolic approximation (Eq. 23) and right) hardness function $\eta(\mathbf{r})$ (Eq. 13) for the $\mathrm{CH}_{2} \mathrm{CHOCH}_{3}$ molecule. 


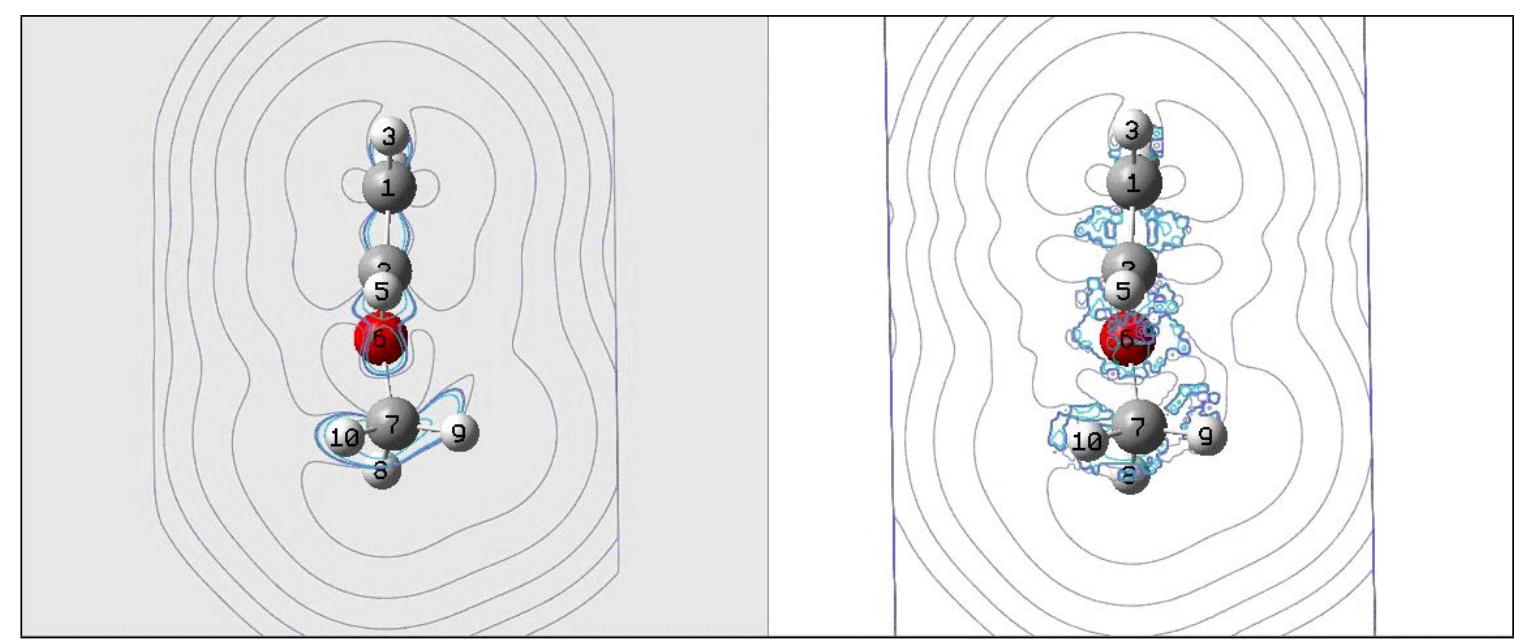

Figure S69. Left) Fukui function $f(\mathbf{r})_{\text {Quadratic expansion }}$ calculated with a parabolic approximation (Eq. 23) and right) hardness function $\eta(\mathbf{r})$ (Eq. 13) for the $\mathrm{CH}_{2} \mathrm{CHOCH}_{3}$ molecule. 
APPENDIX I. Justification of some relationships between parameters of models 1 and 2.

In this appendix, some expressions used in comparisons of the main text will be justified. Starting from the model 1 and truncating from the quadratic term we obtain the expression (A1), this approximation is clearly very rough, for that reason it will only be used to justify the correlations studied in this work.

$$
\delta E(\mathbf{r}) \approx \mu^{0} \cdot \delta \rho(\mathbf{r})+\frac{1}{2} \eta(\mathbf{r}) \delta \rho^{2}(\mathbf{r})
$$

From here we can obtain:

$$
\omega(\mathbf{r}) \approx-\left(\mu^{0} \cdot \delta \rho^{\max }(\mathbf{r})+\frac{1}{2} \eta(\mathbf{r}) \cdot\left(\delta \rho^{\max }(\mathbf{r})\right)^{2}\right)
$$

From model 2, truncating from the first term, we can obtain the expression (A3). This approach is also very crude and, as the previous one, it will only be used to justify the correlations studied in this work.

$$
\delta \rho^{\max }(\mathbf{r}) \approx f(\mathbf{r}) \cdot \Delta N_{\max }
$$

Deriving (A1) we obtain:

$$
\mu \approx \mu^{0}+\eta(\mathbf{r}) \cdot \delta \rho(\mathbf{r})
$$

And from here we get:

$$
0 \approx \mu^{0}+\eta(\mathbf{r}) \cdot \delta \rho^{\max }(\mathbf{r})
$$

And clearing:

$$
\delta \rho^{\max }(\mathbf{r}) \approx-\frac{\mu^{0}}{\eta(\mathbf{r})}
$$

Reordering (A2):

$$
\omega(\mathbf{r}) \approx-\left(\mu^{0} \cdot \delta \rho^{\max }(\mathbf{r})+\frac{1}{2} \eta(\mathbf{r}) \cdot\left(\delta \rho^{\max }(\mathbf{r})\right) \cdot\left(\delta \rho^{\max }(\mathbf{r})\right)\right)
$$

Substituting two terms $\delta \rho^{\max }(\mathbf{r})$ for $f(\mathbf{r}) \cdot \Delta N_{\max }$ and a third for $-\mu^{0} / \eta(\mathbf{r})$ we obtain:

$$
\omega(\mathbf{r}) \approx-\left(\mu^{0} \cdot f(\mathbf{r}) \cdot \Delta N_{\max }+\frac{1}{2} \eta(\mathbf{r}) \cdot\left(f(\mathbf{r}) \cdot \Delta N_{\max }\right) \cdot\left(-\mu^{0} / \eta(\mathbf{r})\right)\right)
$$

and clearing:

$$
\omega(\mathbf{r}) \approx-\frac{1}{2} \mu^{0} \cdot f(\mathbf{r}) \cdot \Delta N_{\max }=\omega \cdot f(\mathbf{r})
$$

Now, we start from: 


$$
\delta \rho^{\max }(\mathbf{r})[\text { model1 }] \approx \delta \rho^{\max }(\mathbf{r})[\operatorname{model} 2]
$$

that is a reasonable starting point. Substituting the expressions $\delta \rho^{\max }(\mathbf{r})$ previously obtained:

$$
-\frac{\mu^{0}}{\eta(\mathbf{r})} \approx f(\mathbf{r}) \cdot \Delta N_{\max }
$$

Reordering:

$$
-\frac{\mu^{0}}{\Delta N_{\max }} \frac{1}{\eta(\mathbf{r})} \approx f(\mathbf{r})
$$

Finally, clearing:

$$
\eta(\mathbf{r}) \approx \frac{\eta}{f(\mathbf{r})}
$$

If we start from (A2) and take into account (A6), replacing and operating we get:

$$
\omega(\mathbf{r}) \approx \frac{\left(\mu^{0}\right)^{2}}{\eta(\mathbf{r})}-\frac{1}{2} \cdot \eta(\mathbf{r}) \cdot \frac{\left(\mu^{0}\right)^{2}}{\eta(\mathbf{r})^{2}}
$$

Simplifying:

$$
\omega(\mathbf{r}) \approx \frac{\left(\mu^{0}\right)^{2}}{2 \eta(\mathbf{r})}
$$

If we combine (A6) and (A2) and we operate we obtain:

$$
\omega(\mathbf{r}) \approx-\left(\mu^{0} \cdot \delta \rho^{\max }(\mathbf{r})+\frac{1}{2}\left(-\frac{\mu^{0}}{\delta \rho^{\max }(\mathbf{r})}\right) \cdot\left(\delta \rho^{\max }(\mathbf{r})\right)^{2}\right)
$$

Finally, simplifying and operating we obtain:

$$
\omega(\mathbf{r}) \approx-\frac{\mu^{0}}{2} \cdot \delta \rho^{\max }(\mathbf{r})
$$


APPENDIX II. Summary of the nomenclature and definitions.

$f(r)$ : Fukui function. It is defined as:

$$
f(r)=\left(\frac{\partial \rho(r)}{\partial N}\right)_{v}
$$

$f_{k}^{+}$: atomic index: condensed-to-atom Fukui function for the " $k$ " atom (nucleophilic attack)

$f_{k}$ : atomic index: condensed-to-atom Fukui function for the " $k$ " atom (electrophilic attack)

$f_{k}^{0}$, atomic index: condensed-to-atom Fukui function for the " $k$ " atom for neutral

(or radical) attack.

$\omega:$ global electrophilicity

$\omega_{k}^{-}:$condensed Philicity to the " $k$ ' atom (electrophilic attack)

$\omega_{k}^{+}$: condensed Philicity to the " $K$ " atom (nucleophilic attack)

$\mu$ : global electronic chemical potential (negative of the electronegativity).

Calculated as:

$$
\mu=\left(\frac{\partial E}{\partial N}\right)_{v}
$$

$\mu_{A}^{0}, \mu_{B}^{0}, \square \quad \mu_{k}^{0}$ : atomic hardness calculated for the neutral molecule.

$\mu_{A}^{+}, \mu_{B}^{+}, \mathbb{} \mu_{k}^{+}$: atomic hardness calculated for the cation. 
$\mu_{A}^{-}, \mu_{B}^{-}, \square \mu_{k}^{-}$: atomic hardness calculated for the anion.

$\eta$ : global chemical hardness. Calculated as:

$$
\eta=\left(\frac{\partial^{2} E}{\partial N^{2}}\right)_{v}
$$

$\Delta N^{+}$: Global charge variation when the neutral molecule turns into a cation.

$\Delta N^{-}$: Global charge variation when the neutral molecule turns into an anion.

$\Delta N_{\max }:$ global maximum amount of electron charge.

$\Delta N_{A}^{\max }$ : atomic maximum amount of electron charge for the $A$ atom.

$\Delta E_{\text {min }}:$ global total energy decrease.

$\epsilon_{H}$ and $\epsilon_{L}$ correspond to the Kohn-Sham one-electron eigenvalues for the

HOMO and the LUMO.

$s(r)$ : softness function. It is defined as:

$$
s(r)=\left(\frac{\partial \rho(r)}{\partial \mu}\right)_{v}
$$

$s_{k}^{\alpha}(\alpha=+,-$ or 0$):$ condensed-to-atom softness (nucleophilic, electrophilic

and attack).

$\Omega_{A}:$ atomic parameter which represents the coefficient of the cubic term in the cubic approximation. It is defined as: 


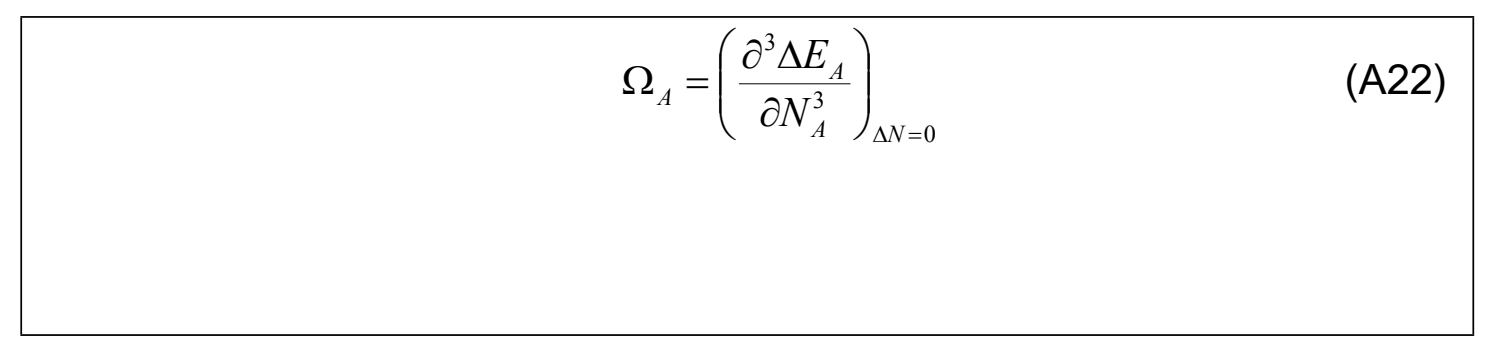

